
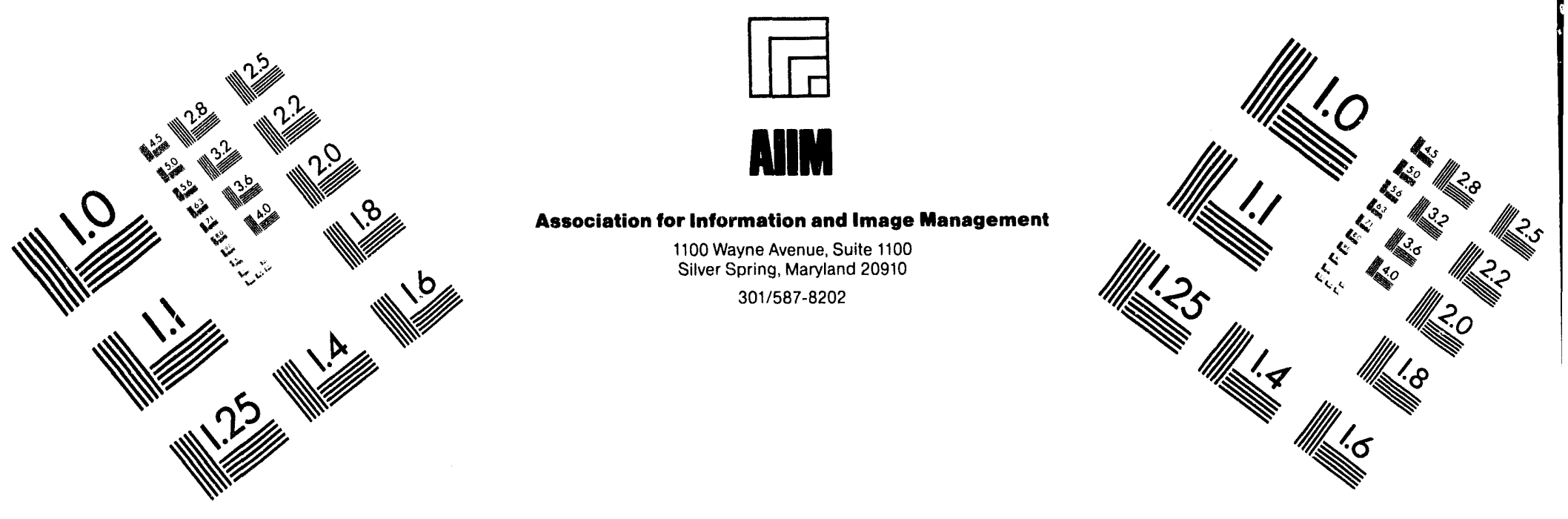

Centimeter

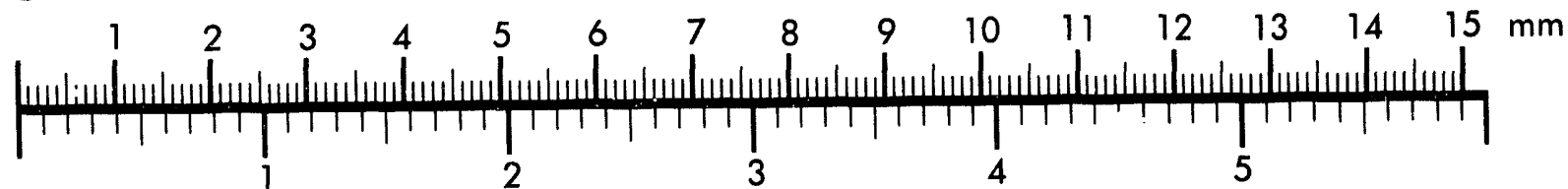
Inches
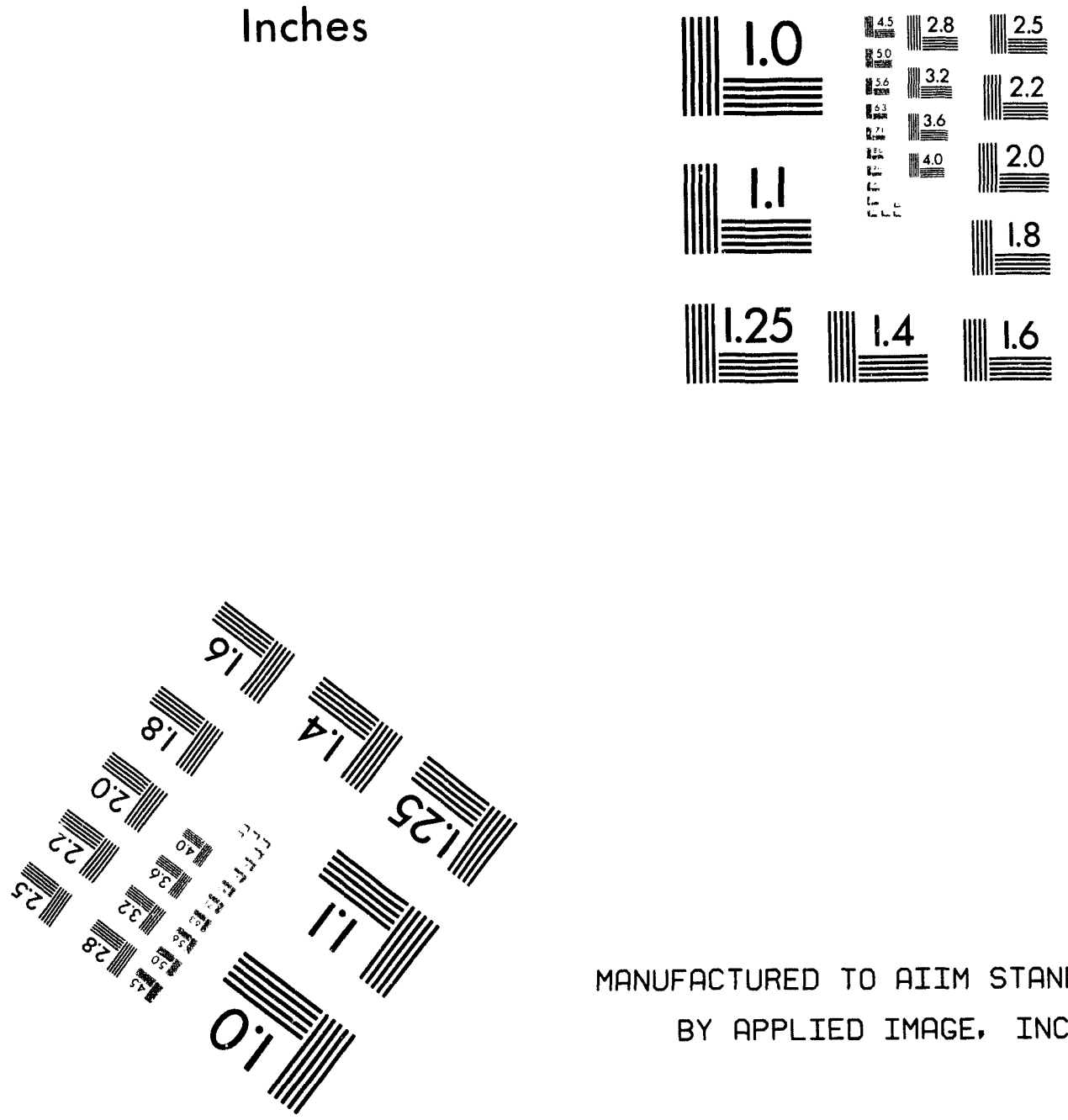

MANUFACTURED TO AIIM STANDARDS

BY APPLIED IMAGE, INC.

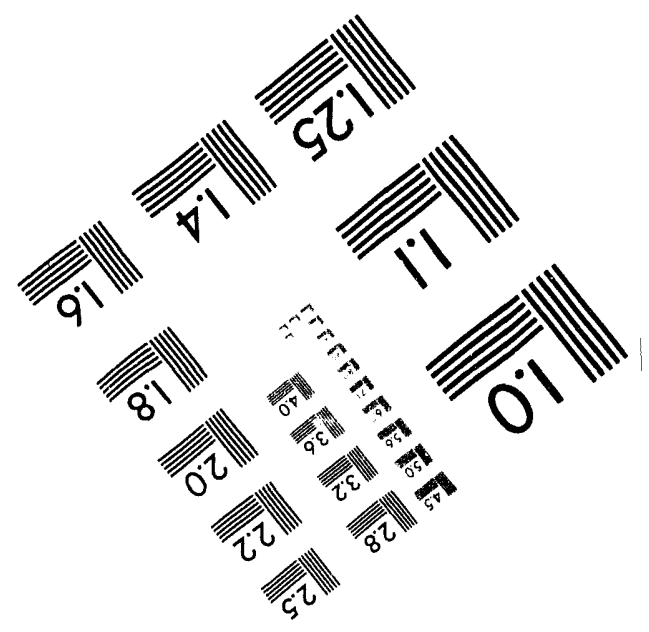



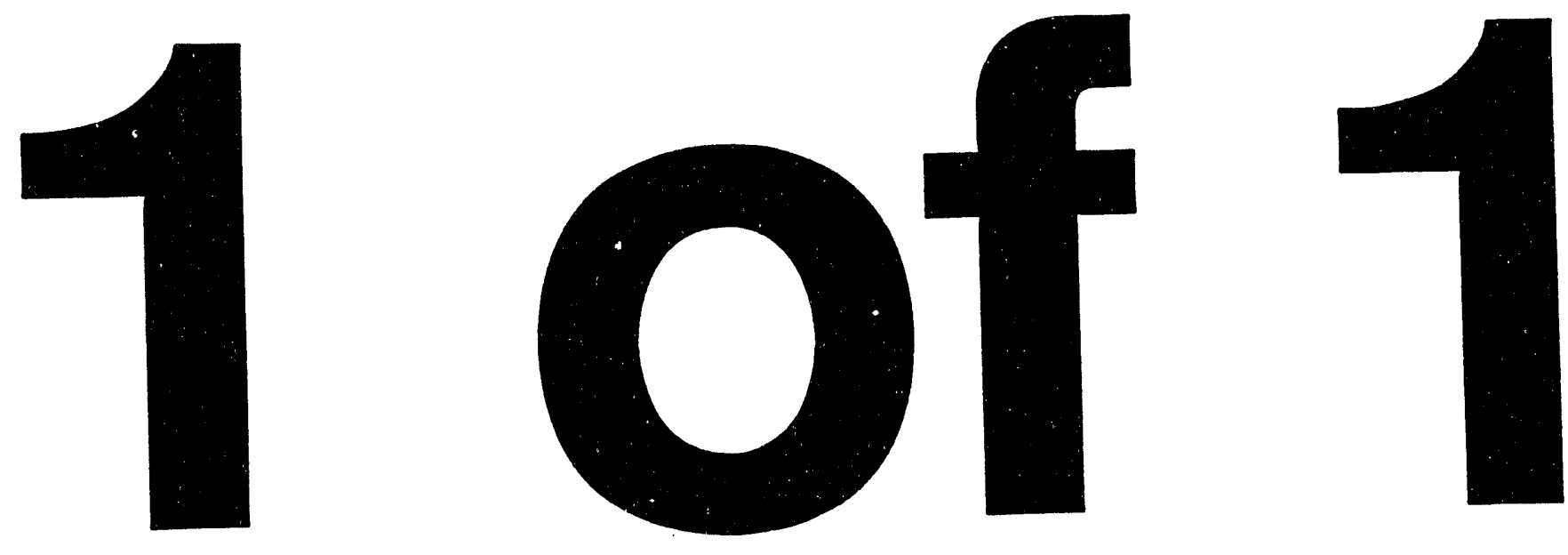


\title{
The Visible Smith-Purcell Radiation Search
}

\author{
R.C. Fernow, H.G. Kirk, S. Ulc and X. Wang \\ Brookhaven National Laboratory \\ Upton, NY 11973
}


The visible Smith-Purcell radiation search

R.C. Fernow, H.G. Kirk, S. Olc \& X. Wang

March 1994

\begin{abstract}
We report here the results of an experiment at the Accelerator Test Facility at Brookhaven National Laboratory to search for the production of visible radiation from the Smith-Purcell effect usirg a $3 \mathrm{MeV}$ electron beam. After running the experiment under a variety of conditions we were unable to isolate a definite signal from Smith-Purcell effect. Any SmithPurcell signal present in the measured radiation was less than $10 \%$ of the background signal.
\end{abstract}




\section{2 / VISIBLE SMITH-PURCELL EXPERIMENT}

\section{Introduction}

Electromagnetic radiation is produced whenever a charged particle passes close to the surface of a metal with a periodic boundary. Smith and Purcell observed this effect for the first time in 1953 by measuring the visible radiation produced by a few hundred keV beam over a grating[1]. Since that time several other experiments using hundred $\mathrm{keV}$ beams have observed visible radiation. The best of these later experiments was by Gover et al [2], who were able to crudely measure the angular dependence of the radiation. The interest in this subject comes from the possibility of using the Smith-Purcell effect to produce a tuneable source of infrared radiation, as a means for particle acceleration, or as a mechanism for a free electron laser.

At the simplest level Smith-Purcell radiation can be pictured as radiation from the oscillating dipole made up of the charged particle and its image charge below the metallic boundary. A simple Huyghens construction for the phase matching between the charged particle and the outgoing wave shows that production of Smithpurcell radiation requires the validity of Eq. 1 (below).

A much more successful model was derived by van den Berg[3], who considered the radiation to come from the evanescent plane waves surrounding the incident particle. Under certain conditions these evanescent waves can diffract from the grating surface and produce real outgoing radiation waves. The same phase matching condition given by Eq. 1 (below) arises in this theory from considerations of wavenumber relations between the Floquet modes of the periodic structure. In addition this theory predicts the observed exponential fall off of the radiated power with the distance of the charged particle from the metallic surface.

In 1987 we proposed an experiment to measure visible radiation from the Smith-Purcell effect using the Accelerator Test Facility (ATF) electron beam [4]. A computer code (GERTY/PERCY) based on the theory of van den Berg was written to calculate the expected amount of radiation. The main goals for the experiment were (1) testing the computer codes for predicting the efficiency of electron-radiation coupling to gratings, (2) to find a Smith-Purcell radiation peak that would be useful as a simple monitor for the grating acceleration experiment, and (3) to obtain experience with stepping motor operation in vacuum and data acquisition that would be useful in designing the grating acceleration experiment.

We felt that the most important new physics contribution from this experiment would be a detailed angular scan, off the plane formed by the electron beam and the normal to the grating (principal plane). A previous experiment [2] with rather coarse data had shown that the radiation pattern had interesting structure off the principal plane. In addition, the Lawson theorem[5] predicts that the radiation from a relativistic electron has to vanish in the 
principal plane. Measurements off the principal plane required two large rotational stages if the detector was to remain a reasonable distance away from the grating.

\section{Beamline}

The experiment was located at the end of the $z$-line at the ATF. There was a stripline and profile monitor located in front of the experiment and a Faraday cup located behind it. The beamline properties were calculated using TRANSPORT. The calculated focal spot [6] had $\sigma_{X}=1.3 \mathrm{~mm}$ and $\sigma_{Y}=0.22 \mathrm{~mm}$ at the grating for $a$ momentum spread $\delta \mathrm{p} / \mathrm{p}=0.2 \%$. Most of the data was taken at a beam momentum of $2.8 \mathrm{MeV} / \mathrm{c}$. Unfortunately, the last quad in the $z$-line before the experiment was a SLAC quad. This quad is more suited to a higher energy beamline. In our case it had to be run with a very low current and thus we were susceptible to errors caused by the residual field.

\section{Data acquisition system}

The data acquisition system was designed around a CAMAC crate connected to a PC. NIM electronics were used in the trigger system. The major components were:

(1) CAMAC/PC interface, DSP Technology p/n 6001, PC004.

(2) Stepping Motor Controller, DSP Technology p/n E500.

(3) Charge sensitive ADC, LeCroy Corp. $\mathrm{p} / \mathrm{n} 2249 \mathrm{~A}$.

(4) Thermocouple readout, Kinetic Systems $\mathrm{p} / \mathrm{n} 3525,1991$.

There were three stepping motor channels used, two for the rotational orientation of the optical detector and one for the height of the grating relative to the electron beam position. The large rotating stage had a resolution of 60 steps per degree, the small rotating stage 120 steps per degree, and the linear stage 0.1 $\mu \mathrm{m}$ per step. The major tasks of the computer software were to

(1) initialize stepping parameters and data files

(2) check that requested moves would not result in damage to the collection system

(3) move the rotational stages so that the detector was at the desired orientation

(4) read the $A D C$ values for the optical detector, Faraday cup, striplines and any other diagnostics after each beam pulse 


\section{4 / VISIBLE SMITH-PURCELL EXPERIMENT}

(5) check for motor heating in the vacuum using the thermocouples.

The program had a SCAN mode to collect data automatically over given angular ranges, along with statistical analyses and a graphics display.

\section{Gratings}

Three gratings were used in the experiment.

(1) Edmunds Scientific p/n B43.004, $12.5 \times 25 \mathrm{~mm}, 1200$ grooves per $\mathrm{mm}, 500 \mathrm{~nm}$ blaze wavelength, ruled visible grating.

(2) Edmunds Scientific p/n B43.215, $12.5 \times 25 \mathrm{~mm}, 1200$ grooves per $\mathrm{mm}$, holographic visible grating.

(3) A $10.6 \mu \mathrm{m}$ period grating made by John Warren in the Instrumentation Division according to the design of Ref. [7].

The commercial gratings had a period of $833 \mathrm{~nm}$. The ruled gratings were made by cutting in a layer of epoxy plastic on top of a glass substrate. The plastic was then coated with a thin layer of aluminum. The sinusoidal profile on the holographic grating was made by interfering two laser beams above the plastic. The $10 \mu \mathrm{m}$ period grating was etched out of silicon and coated with a thin layer of aluminum.

We had a set of narrow bandwidth optical filters for wavelength selection. The wavelength $\lambda$ of Smith-Purcell radiation is related to the grating period $D$ by

$$
\lambda=\frac{D}{m}\left(\frac{1}{\beta}-\sin \eta\right)
$$

where $\beta$ is the relativistic factor, $m$ is the radiation order and $\eta$ is the angle shown in Fig. 1. Table 1 shows the expected emission angles for a momentum of $2.8 \mathrm{MeV} / \mathrm{C}$, which corresponds to the relativistic factors $\gamma=5.57$ and $\beta=0.984$.

\begin{tabular}{|c|c|c|c|c|c|c|}
\hline & le 1 & ission & gles $f$ & commerc & \multicolumn{2}{|c|}{ gratings } \\
\hline & \multicolumn{6}{|c|}{$\lambda \quad[\mathrm{nm}]$} \\
\hline $\mathrm{m}$ & 400 & 500 & 600 & 700 & 800 & 900 \\
\hline 1 & 32.4 & 24.6 & 17.2 & 10.1 & 3.2 & -3.7 \\
\hline 2 & 3.2 & -10.6 & -25.1 & -41.6 & -64.7 & \\
\hline 3 & -25.1 & -51.6 & & & & \\
\hline
\end{tabular}


VISIBLE SMITH-PURCELL EXPERIMENT / 5

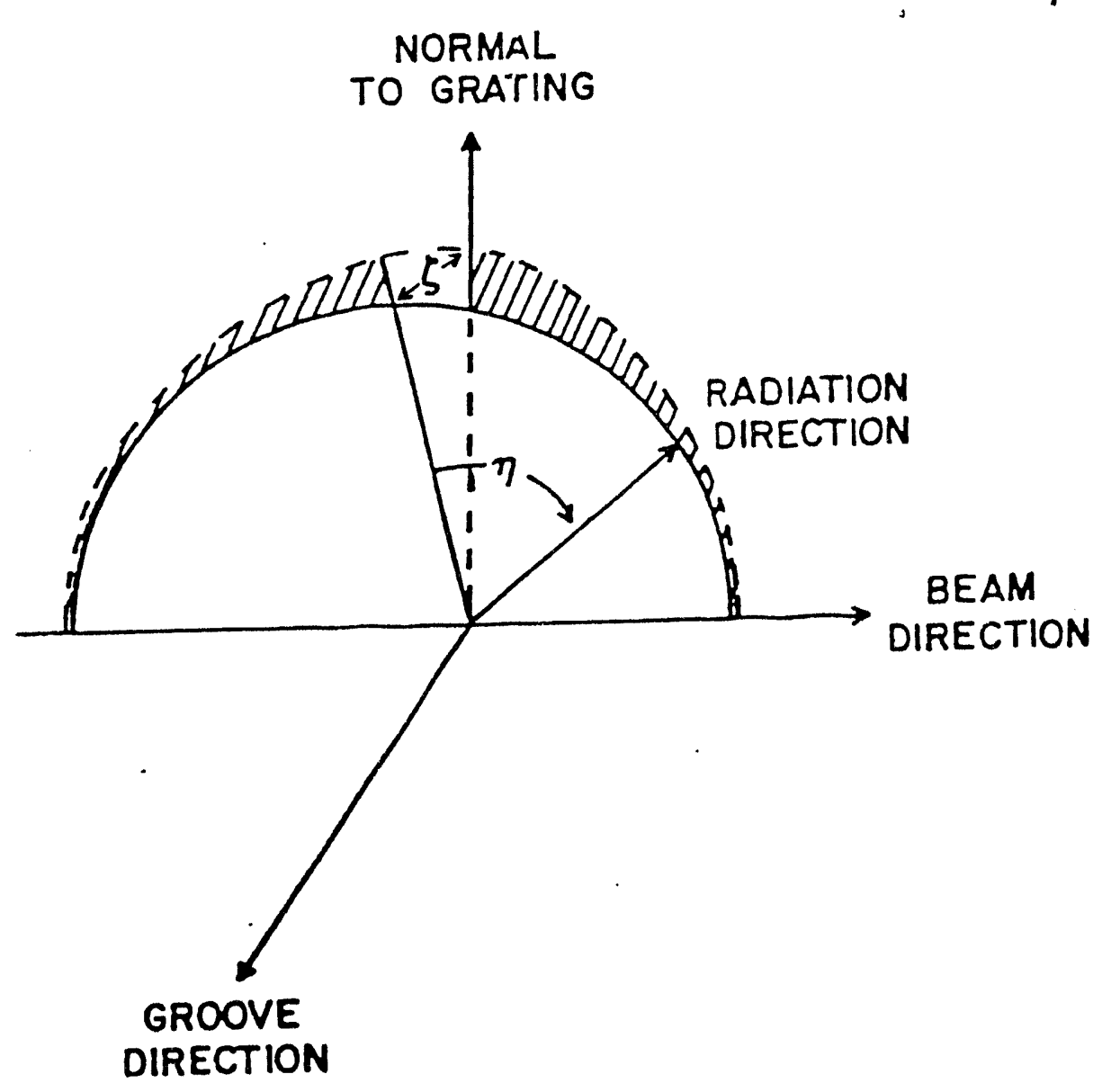

Fig. 1 Definition of radiation emission angles, $\eta$ and $\zeta$. 


\section{6 / VISIBLE SMITH-PURCELL EXPERIMENT}

\section{Experimental configuration (1991)}

The first experimental configuration is shown in Fig. 2. The experiment was separated from the ATF beamline by a $16 \mu \mathrm{m}$ thick Be window. The window was located as close as possible to the grating by using a beam "snout" inside the vacuum chamber.

The radiation detector was a Hamamatsu model R1414 PMT inside the vacuum chamber. A narrow bandwidth optical filter was used in front of the PMT for wavelength selection. The collection angle acceptance was 5 millisteradian. A lead foil was wrapped around the PMT and a lead-glass filter was mounted in front of it to reduce the $\mathrm{x}$-ray backgrour.d. The photocathode opening on the tube was $4 \mathrm{~mm}$ $x 13 \mathrm{~mm}$. The PMT was located $10 \mathrm{~cm}$ from the center of the grating. An LED and a light bulb with adjustable current were provided to check the PMT calibration in situ.

A fixed molybdenum beam scraper was used in front of the grating. The top surface of the scraper was at the nominal beam height. The scraper was $2.54 \mathrm{~mm}$ high and $8.13 \mathrm{~mm}$ deep along the beam direction. It was electrically insulated from the rest of the apparatus by a piece of plastic, so that the collected charge could be used as a beam diagnostic.

The grating was mounted on the vertical translation stage immediately after the scraper, as shown in Fig. 3. The electron beam then passed through the hollow shaft of the large rotating stage and into a Faraday cup mounted outside the experiment chamber.

The quality of the grating groove profiles was checked by illuminating the surfaces with a green HeNe laser. Fig. 4 shows a portion of the spectrum from the $10 \mu \mathrm{m}$ grating and from one of the commercial gratings. Spectral orders are clearly seen indicating that the gratings at least began the experiment with a good surface profile.

We used the ATF beam on a total of 23 days in 1991. We fixed problems due to noise from the modulator and stepping motors, misalignment in the $z$ line, timing jumps, and saturation of the PMT signal. We studied the dependence of the signal on beam tune, trim coil steering, and grating height.

On two occasions the beam profile at the location of the grating was measured in special runs with a phosphor and camera inside the experiment chamber. The beam was rectangular with a nominal vertical height of $\approx 200 \mu \mathrm{m}$. 


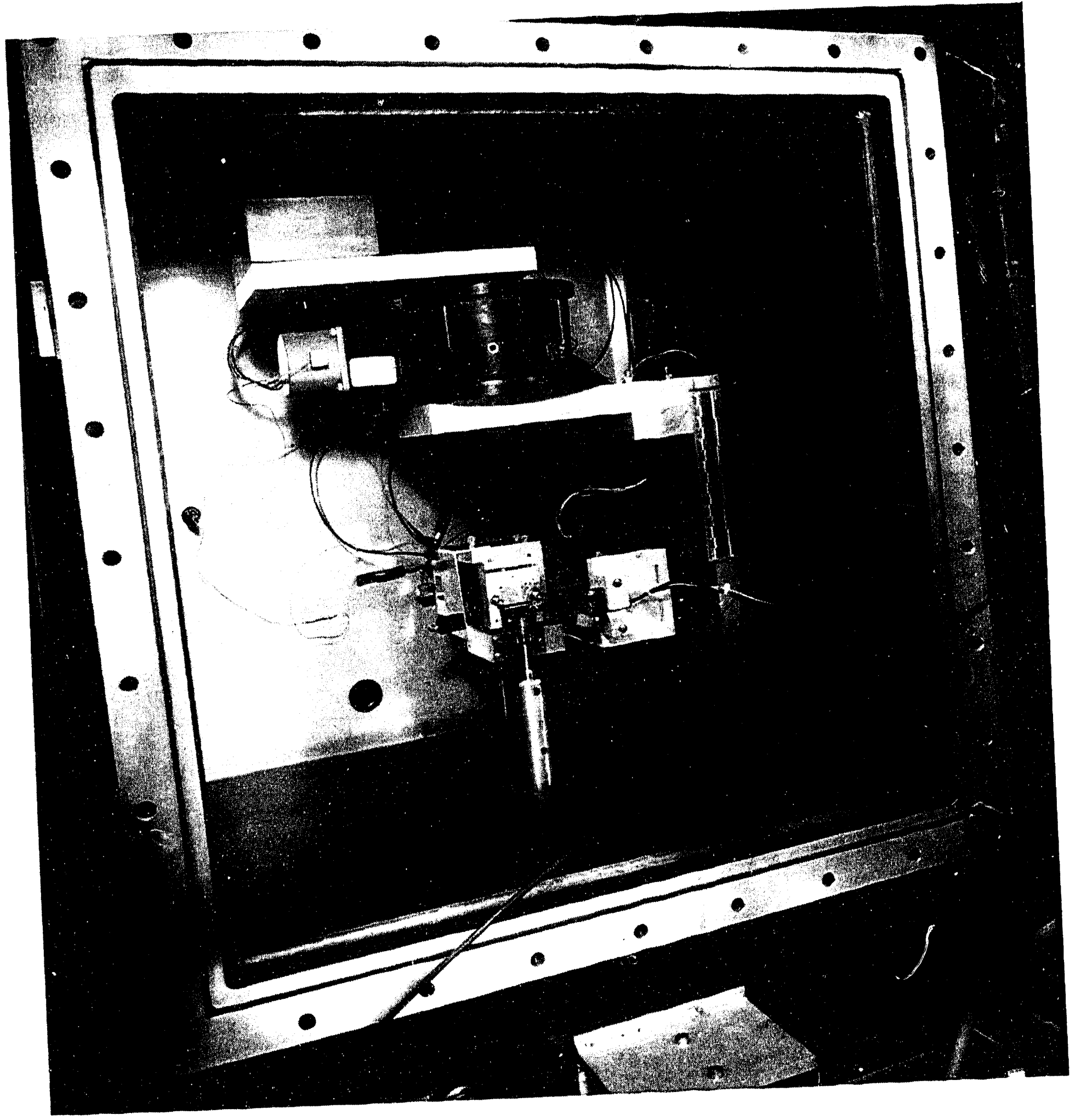

Eig. 2 The interior of the experiment chamber for the 1991 run. 


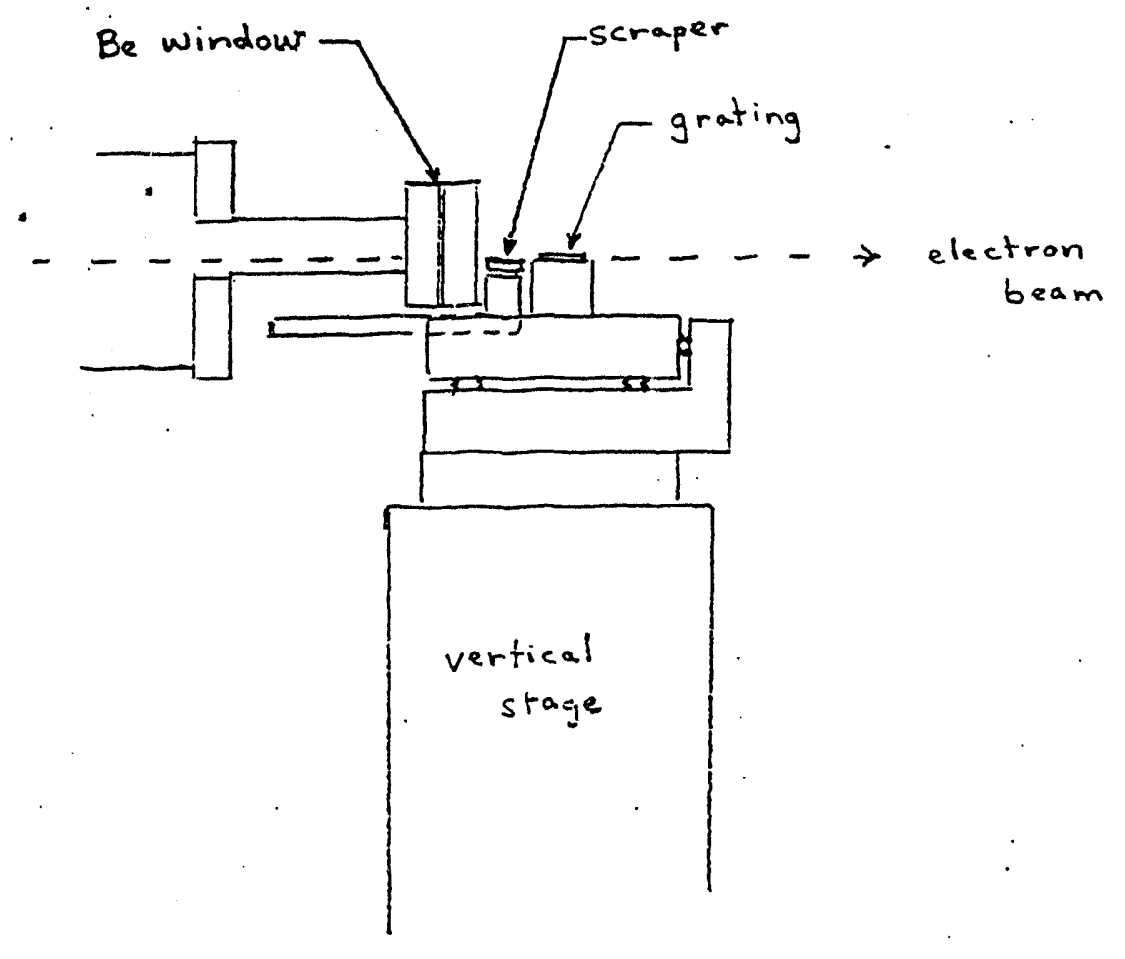

Fig. 3 Details of the experimental arrangement in the vicinity of the grating. 
VISIBLE SMITH-PURCELL EXPERIMENT / 9
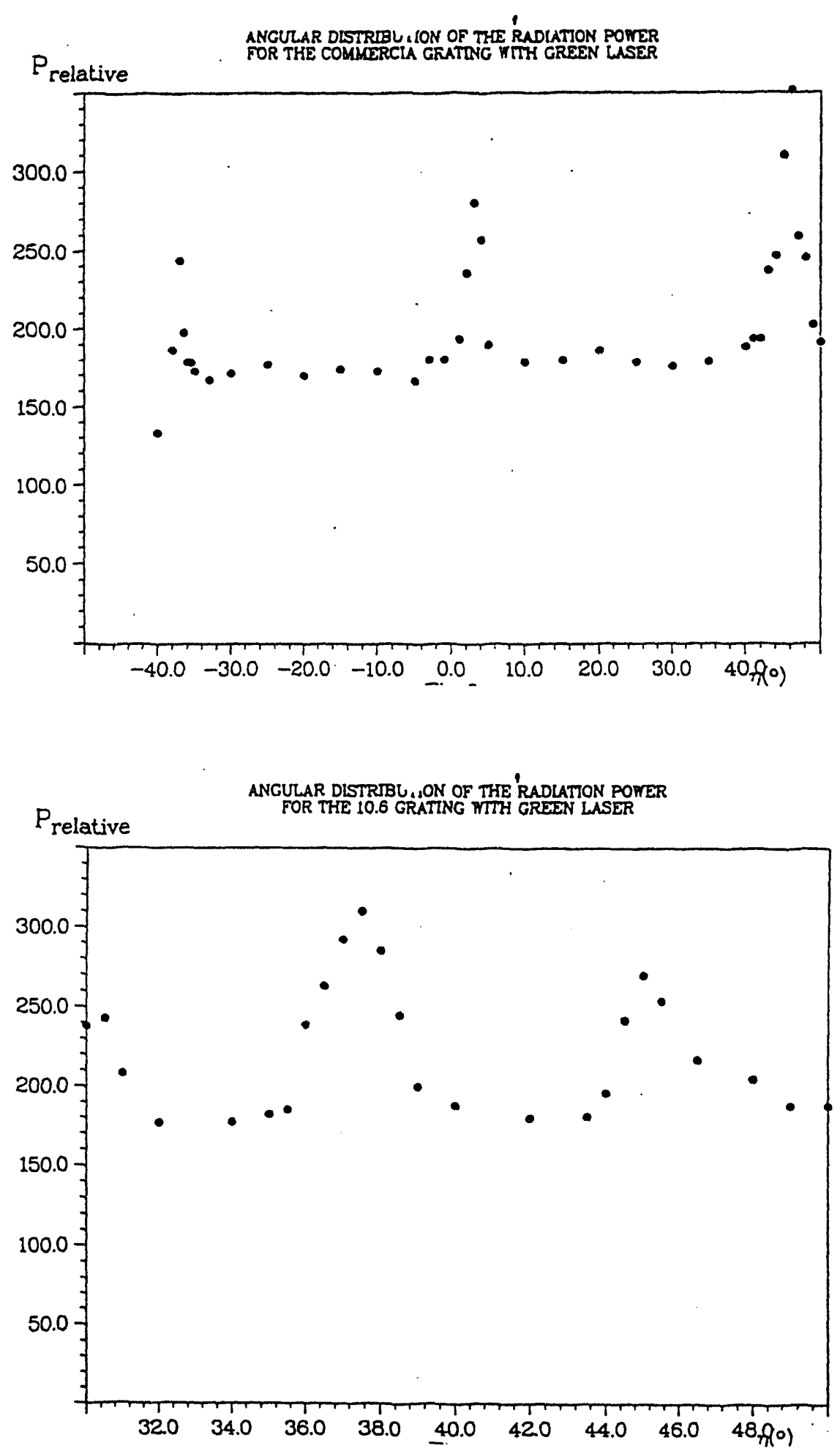

Fig. 4 Angular distribution of laser light diffracted from the commercial and $10 \mu \mathrm{m}$ gratings. 


\section{0 / VISIBLE SMITH-PURCELL EXPERIMENT}

Fig. 5 shows some of the calibration data for the SLAC quad and the horizontal and vertical trim magnets that were performed before most runs. These measurements were made by measuring the charge collected on the scraper. The SLAC quad was scanned to check that the maximum signal occurred at the nominal current. This was interpreted to mean that the focal point was still at the center of the grating. Then a scan was made using the vertical trim coil.

When the beam is steered down no charge will be collected on the scraper. Then, as the beam height continues to increase, some of the beam begins to intercept and the collected charge should increase. The charge is maximum when the beam is centered on the front edge of the scraper. Then the collected charge begins to decrease again as the beam goes over the top edge of the scraper. We chose as a typical set point the half-way point on the upper edge. Finally, a scan was made using the horizontal trim coil. Since the beam spot was wide and the grating surface was also wide, this was usually not very sensitive.

Scans were made of the optimal height of the grating with respect to the fixed height of the scraper and the current location of the electron beam. Fig. 6 shows typical forward-backward scans for three grating heights. Each step corresponded to $\approx 0.1 \mu \mathrm{m}$.

Smith-Purcell radiation from the commercial grating and using the $400 \mathrm{~nm}$ optical filter should have occurred at $\eta=\{31,2,-26\}$ for orders $\{1,2,3\}$. Scans were frequently taken of the angle $\}$ for these three values of $\eta$. Typical data are shown in Fig. 7. There is no simple prediction from the theory on how the radiation intensity should vary with $\zeta$, but an earlier experiment [2] had seen an enhancement in this angle.

Most of the 1991 data was taken using dark current from the electron gun. The radiation was integrated over periods of 0.6 to $1 \mu \mathrm{s}$. Occasional runs were made with photocurrent when the YAG laser system was available and operating with reasonable stability. Super charge was discovered at the ATF right at the end of the 1991 run.

We show in Fig. 8 scans of the angle $\eta$ for dark current, photocurrent, and supercharge. Here one is looking for peaks at $\eta$ $\approx\{31,2,-26\}$. No reproducible, statistically significant peaks were observed. 

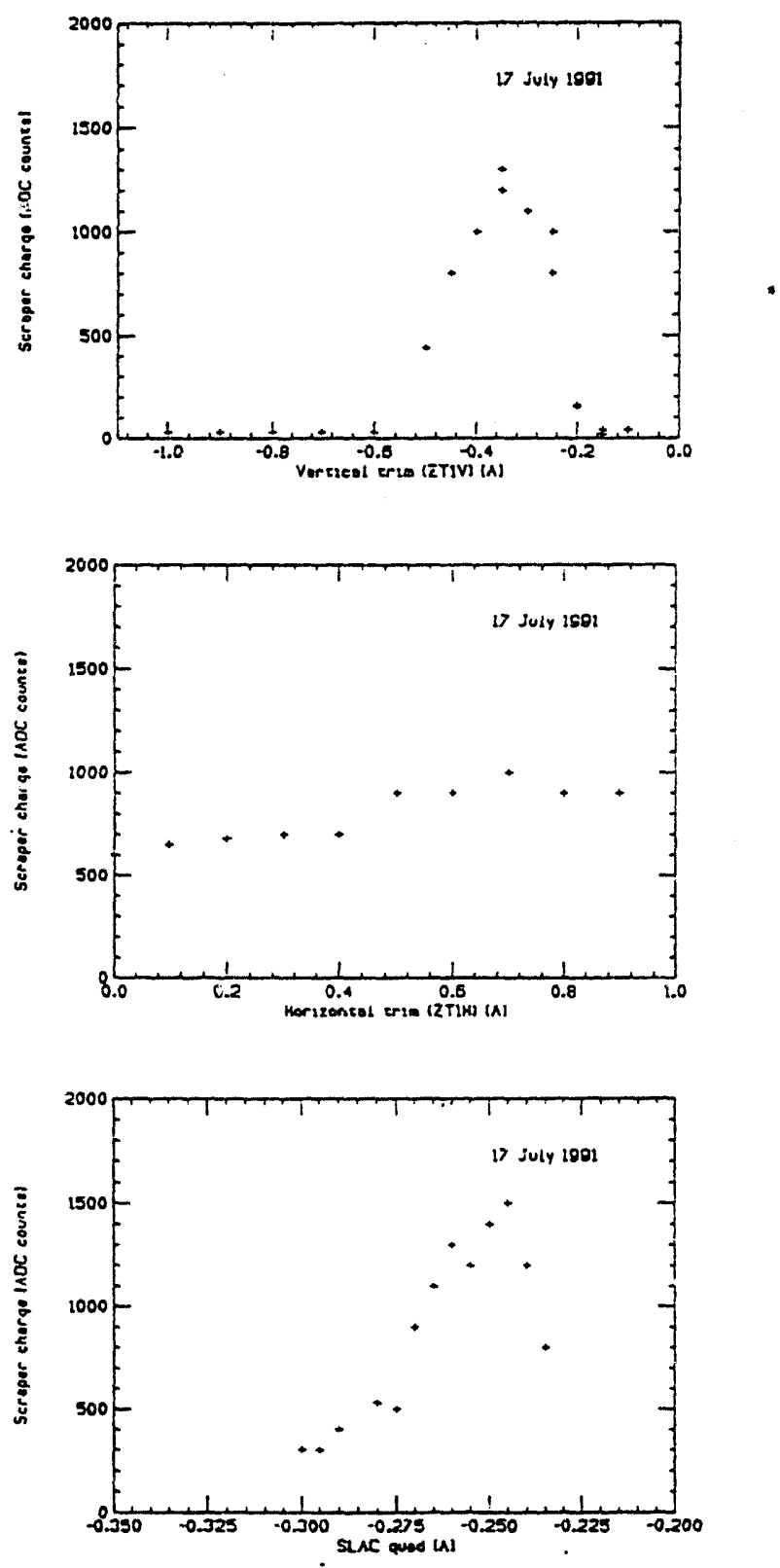

Fig. 5 Variation in scraper charge due to changes in vertical trim coil, horizontal trim coil, and SLAC quadrupole current. 

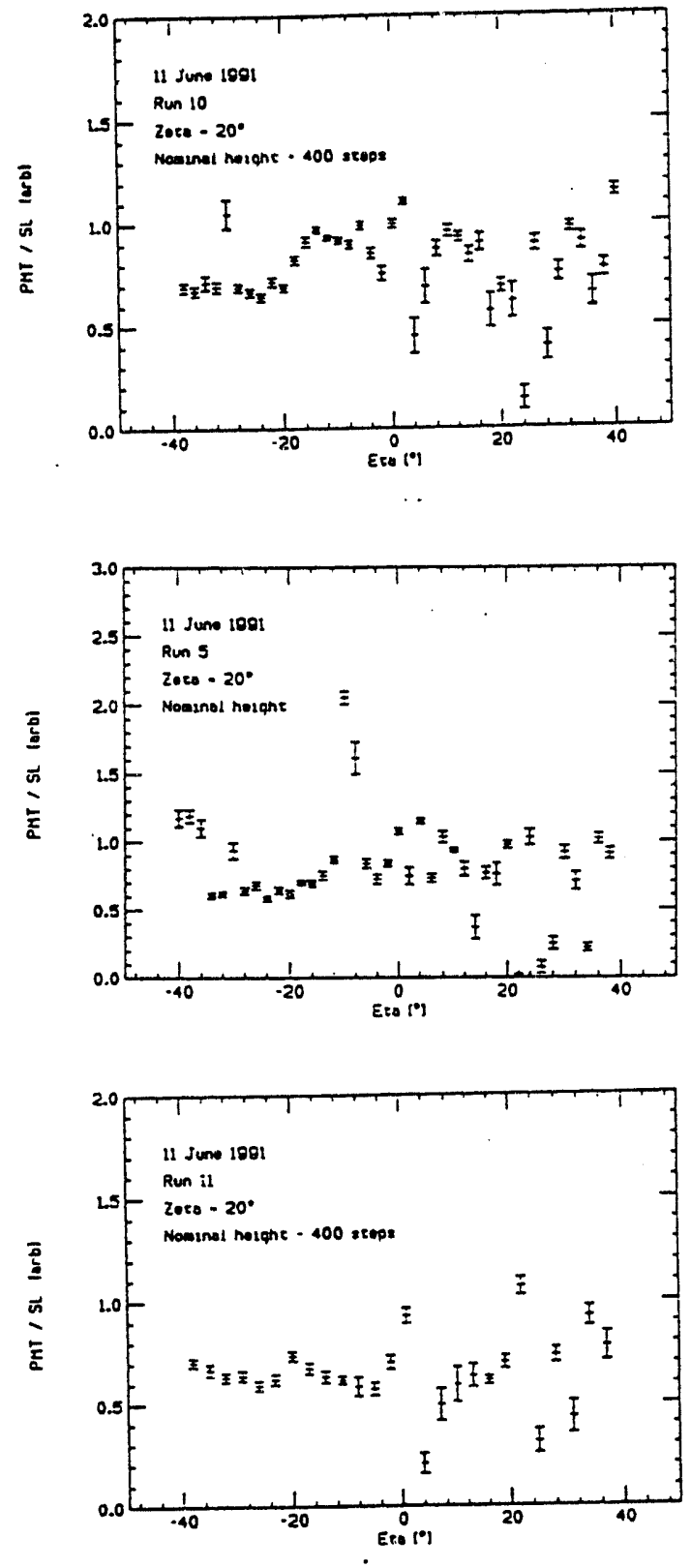

Fig. 6 Normalized PMT signal as a function of $\eta$ for three different grating heights with respect to the beam. 


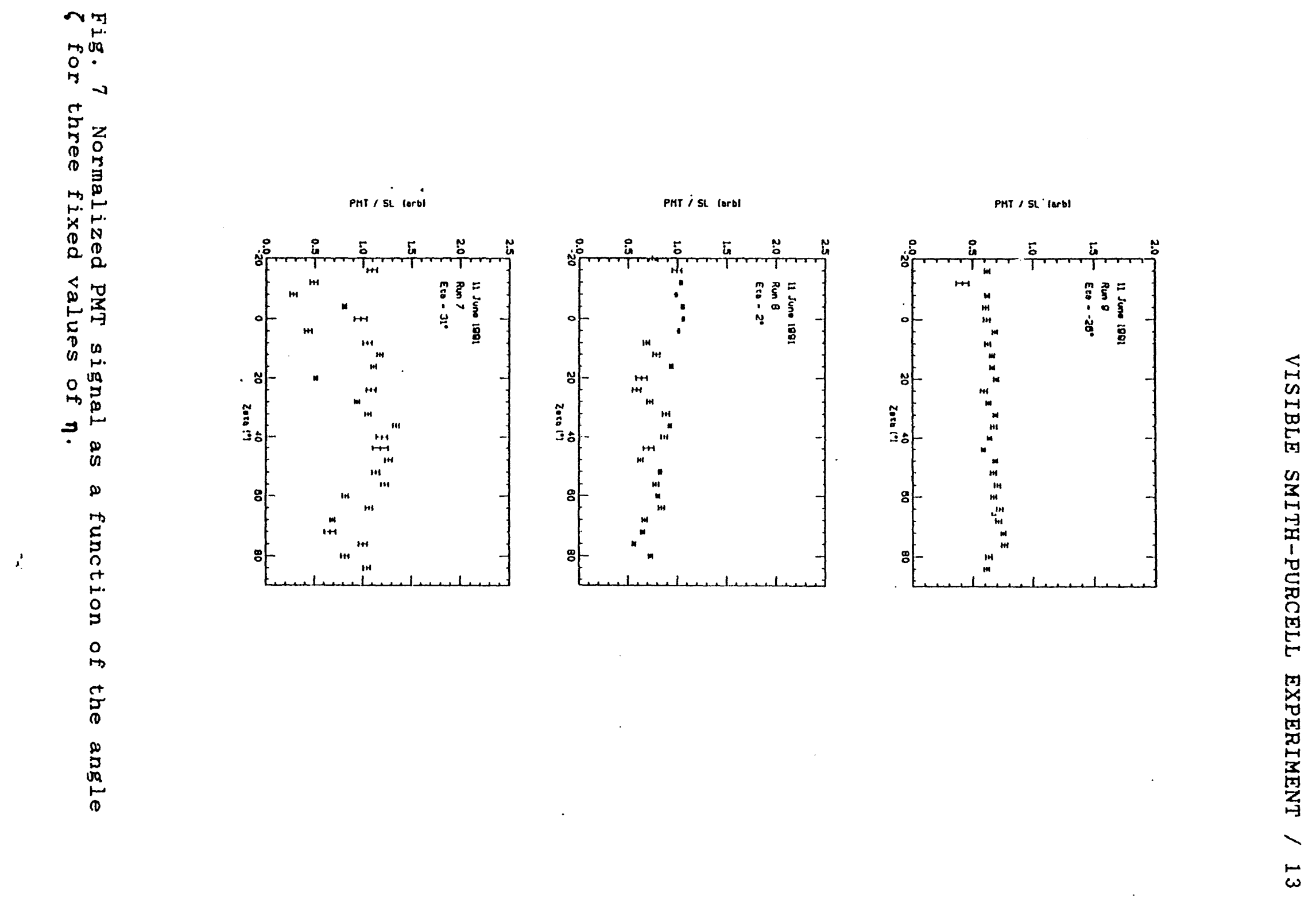



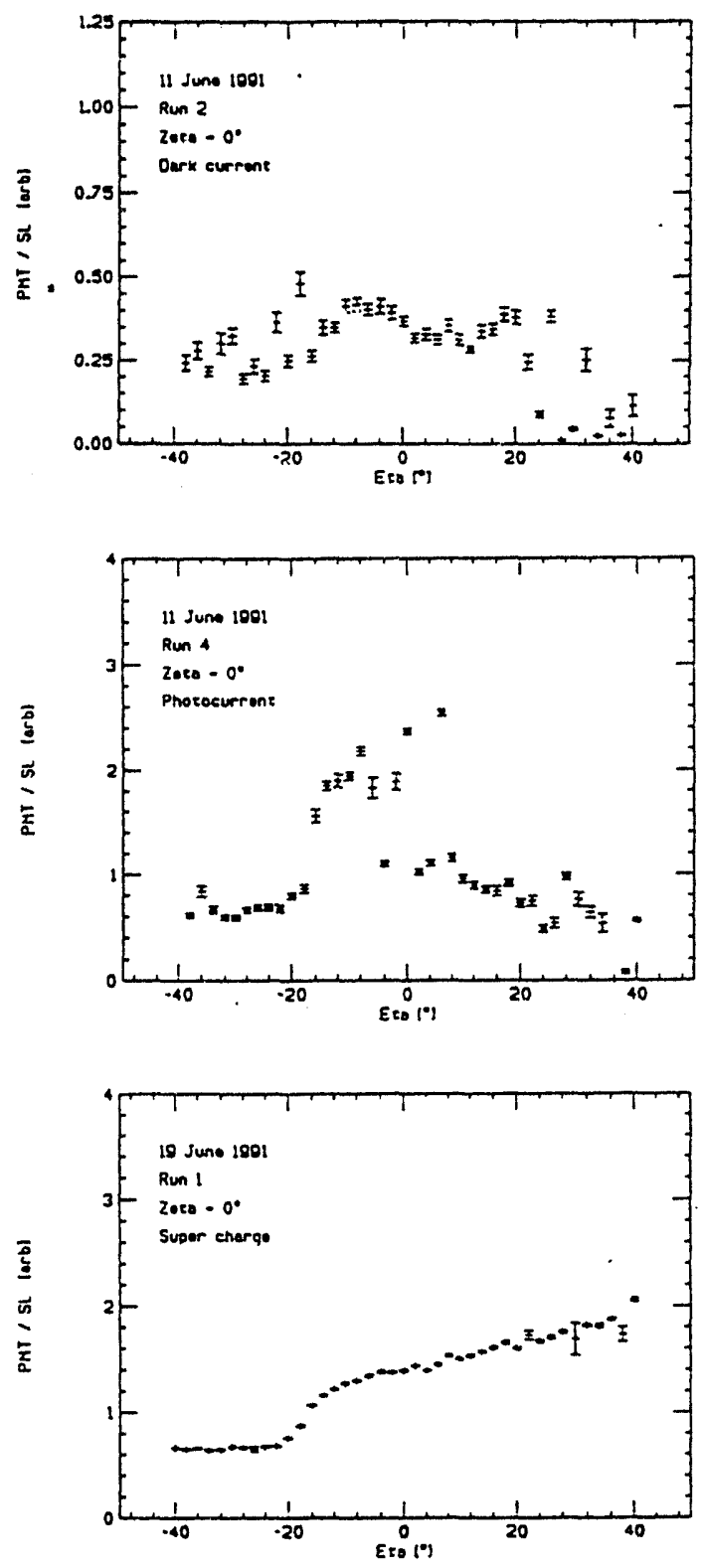

Fig. 8 Normalized PMT signal as a function of the angle $\eta$ for dark current, photocurrent, and super charge. 


\section{Experimental configuration (1992)}

It was felt that much of the potentially useful electron beam in the 1991 runs was being lost due to scattering in the Be window [8]. For this reason the new configuration shown in Fig. 9 was adopted. The grating was placed inside a high vacuum glass tube at the end of the ATF beam line, as shown in Fig. 10. The grating rested on a stainless steel holder. Unfortunately, it was impossible to use a vertical translation stage with the grating in this configuration. The exterior exit end and bottom of the glass tube were coated with conductive paint to help remove any charge build-up on the glass.

In 1991 the PMT was being subjected to a large background of $x$ rays. The $x$ ray background was reduced by removing the beam scraper and moving the PMT into a shielded box outside the vacuum chamber. A new PMT (Hamamatsu R666) was used, which had enhanced long wavelength responsivity in the red to near-infrared portion of the spectrum. The radiation was collected using a new lens assembly coupling into an optical fiber placed on the rotating stages.

In this configuration we lost the ability to use the scraper as a beam alignment diagnostic. In its place a new Faraday cup shown in Fig. 11 was constructed and mounted directly downstream of the glass tube. The Faraday cup had a measured sensitivity including cable capacitance of $10 \mathrm{mV} / \mathrm{pC}$.

The ATF beam was used for a total of 14 days in 1992. Most of the data was taken using super charge and gate widths around 100 ns. The beam charge per pulse was typically $\approx 200 \mathrm{pC}$. We continued to examine radiation from the commercial gratings for the first 4 of these days. Fig. 12 shows some examples of $\zeta$ scans. Fig. 13 shows $\eta$ scans for five different wavelengths. None of the expected peaks in Table 1 are observed. 


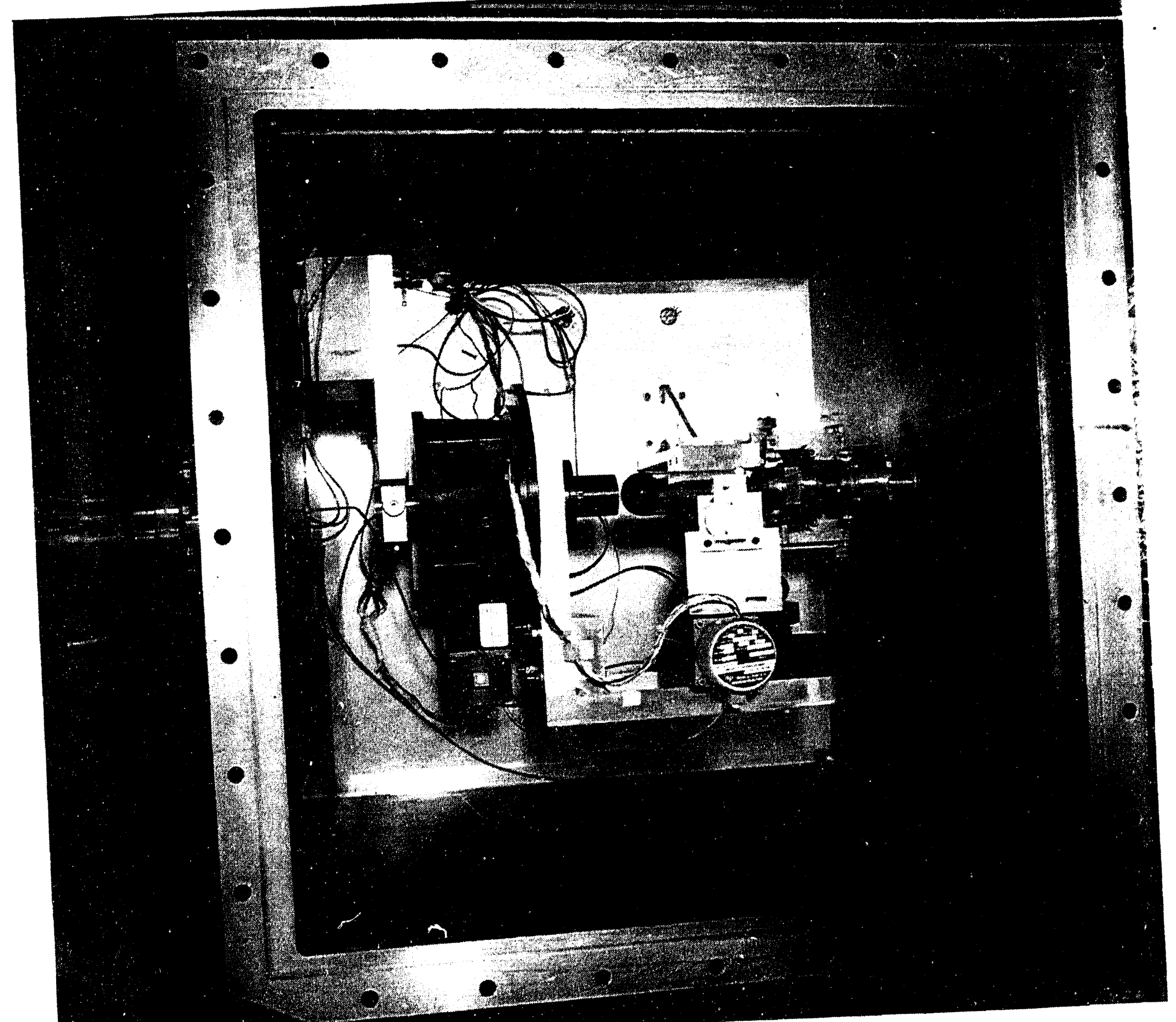

Fig. 9 Interior of the experimental chamber used in the 1992 data runs. 


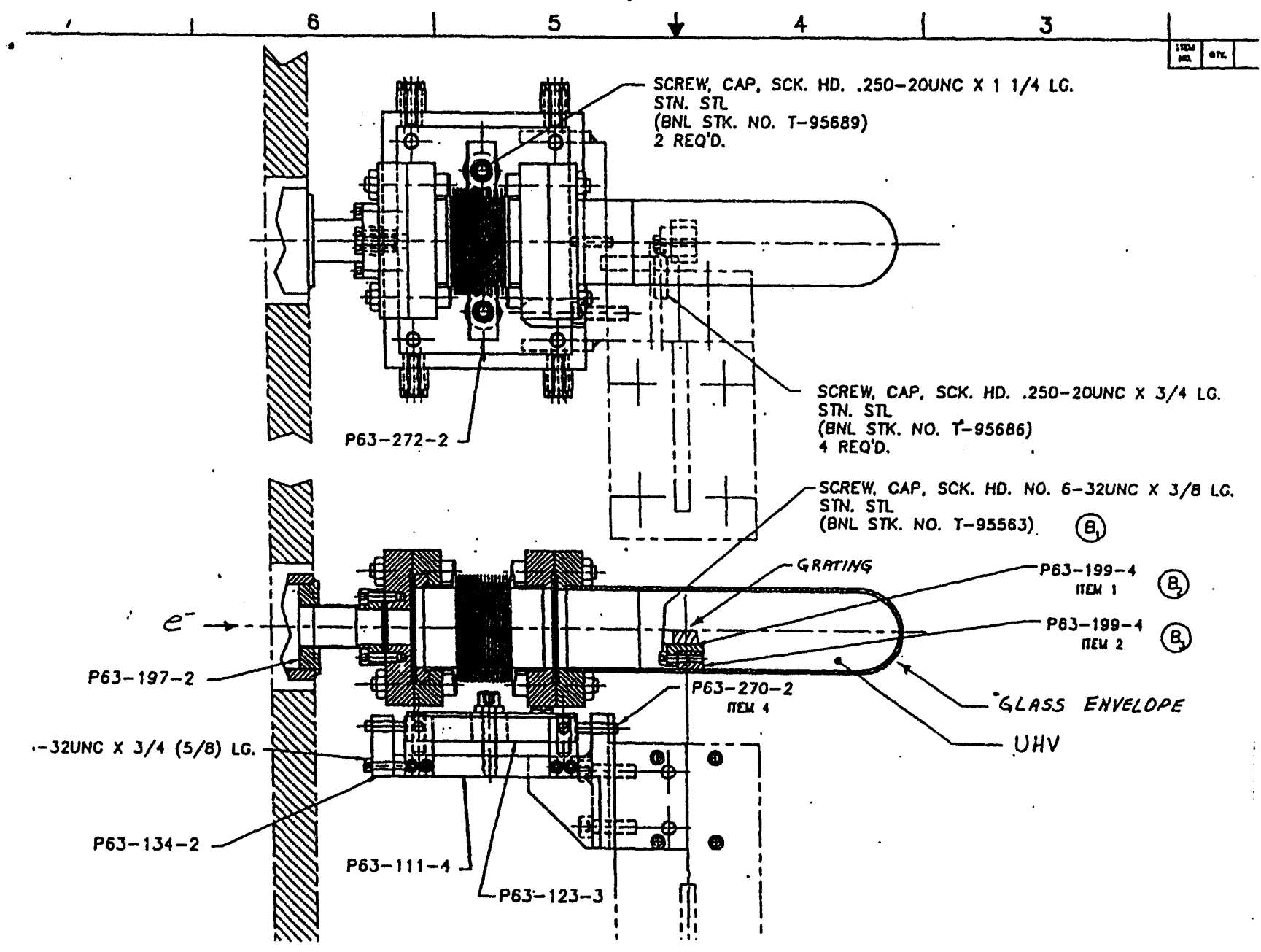

Fig. 10 Details of the grating and glass beam extension tube. 
$18 /$ VISIBLE SMITH-PURCELL EXPERIMENT

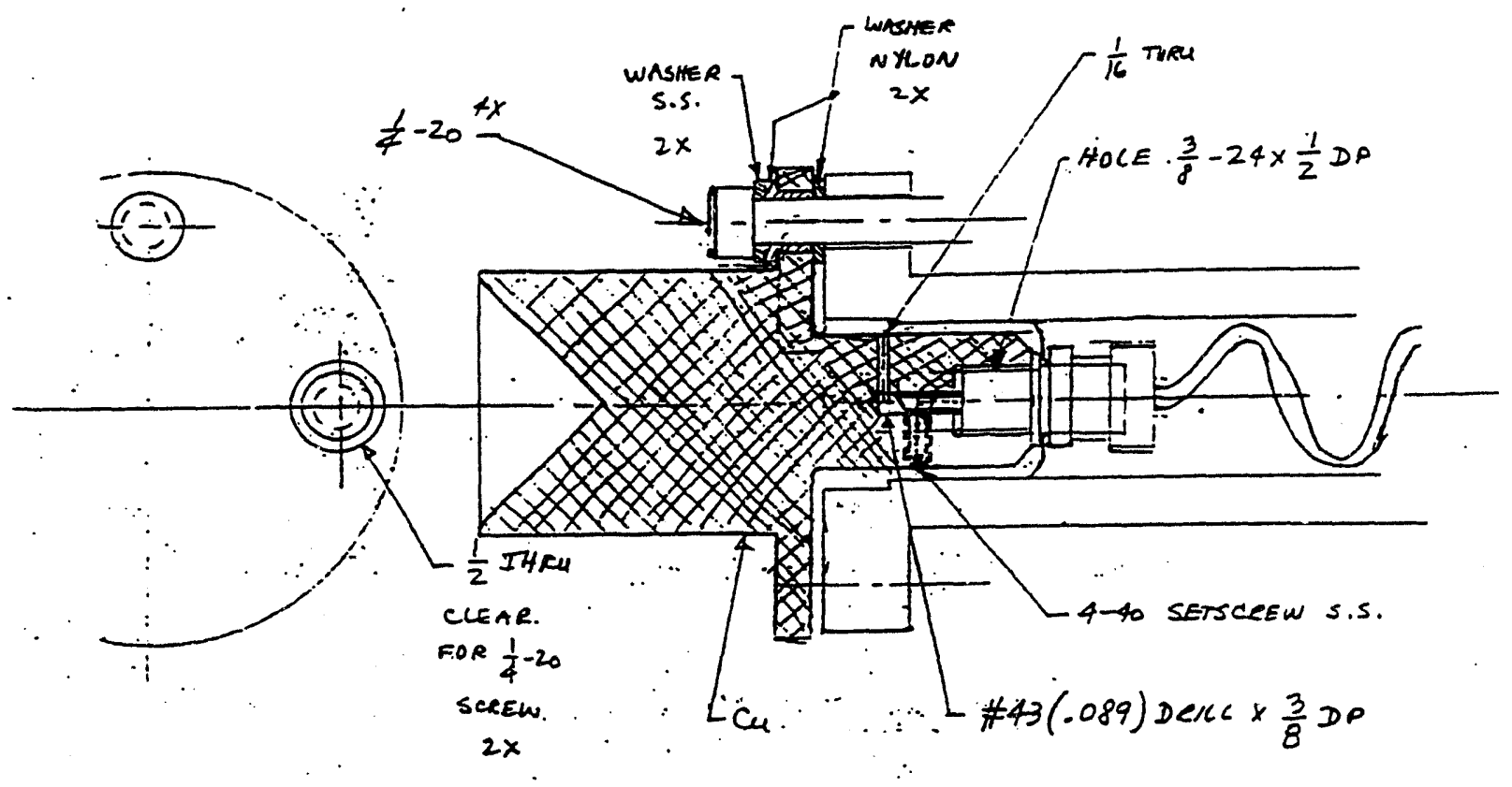

Fig. 11 Details of new Faraday cup detector. 

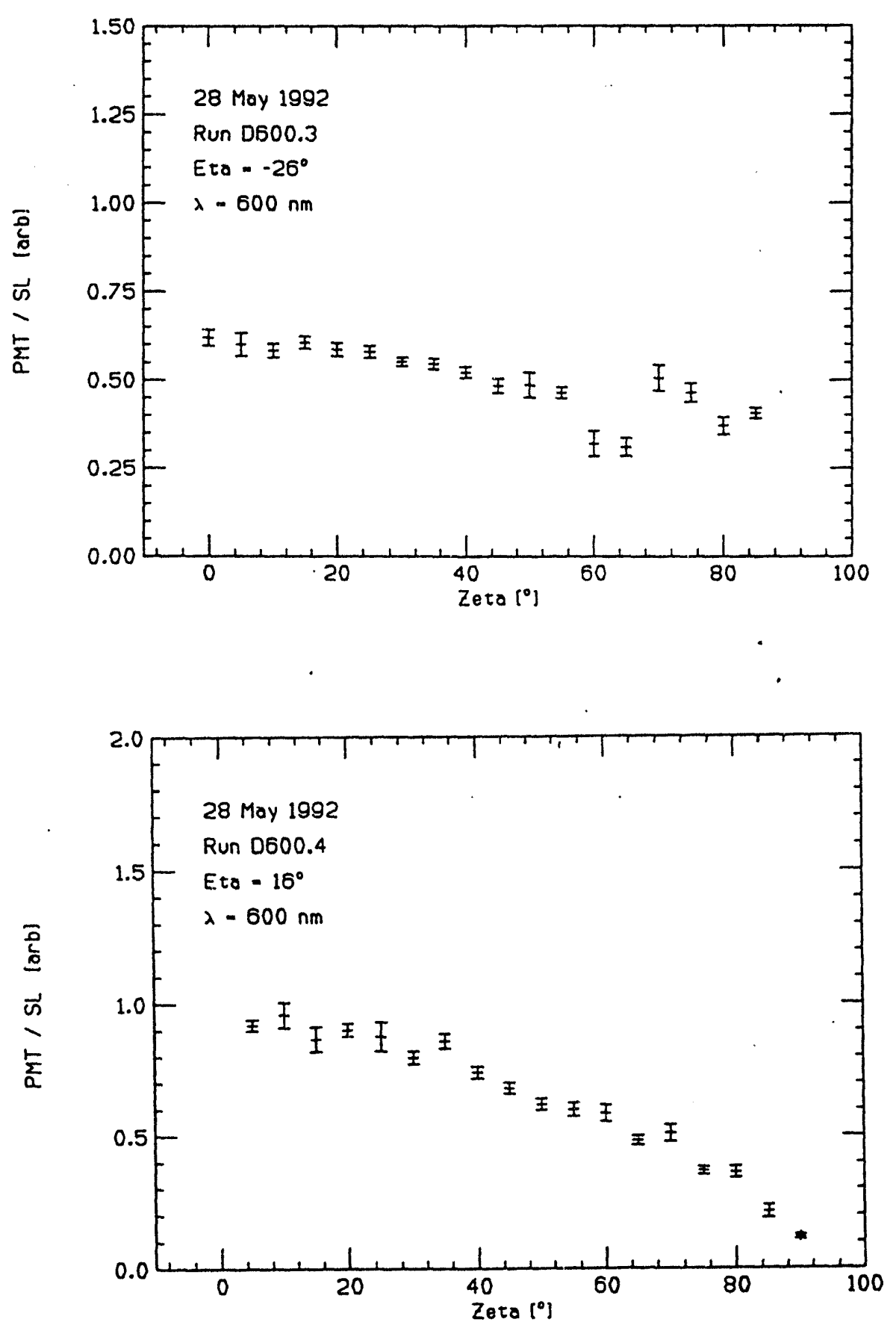

Fig. 12 Normalized PMT signal from the commercial grating as a function of the angle $\zeta$ for two fixed values of the angle $\eta$. 

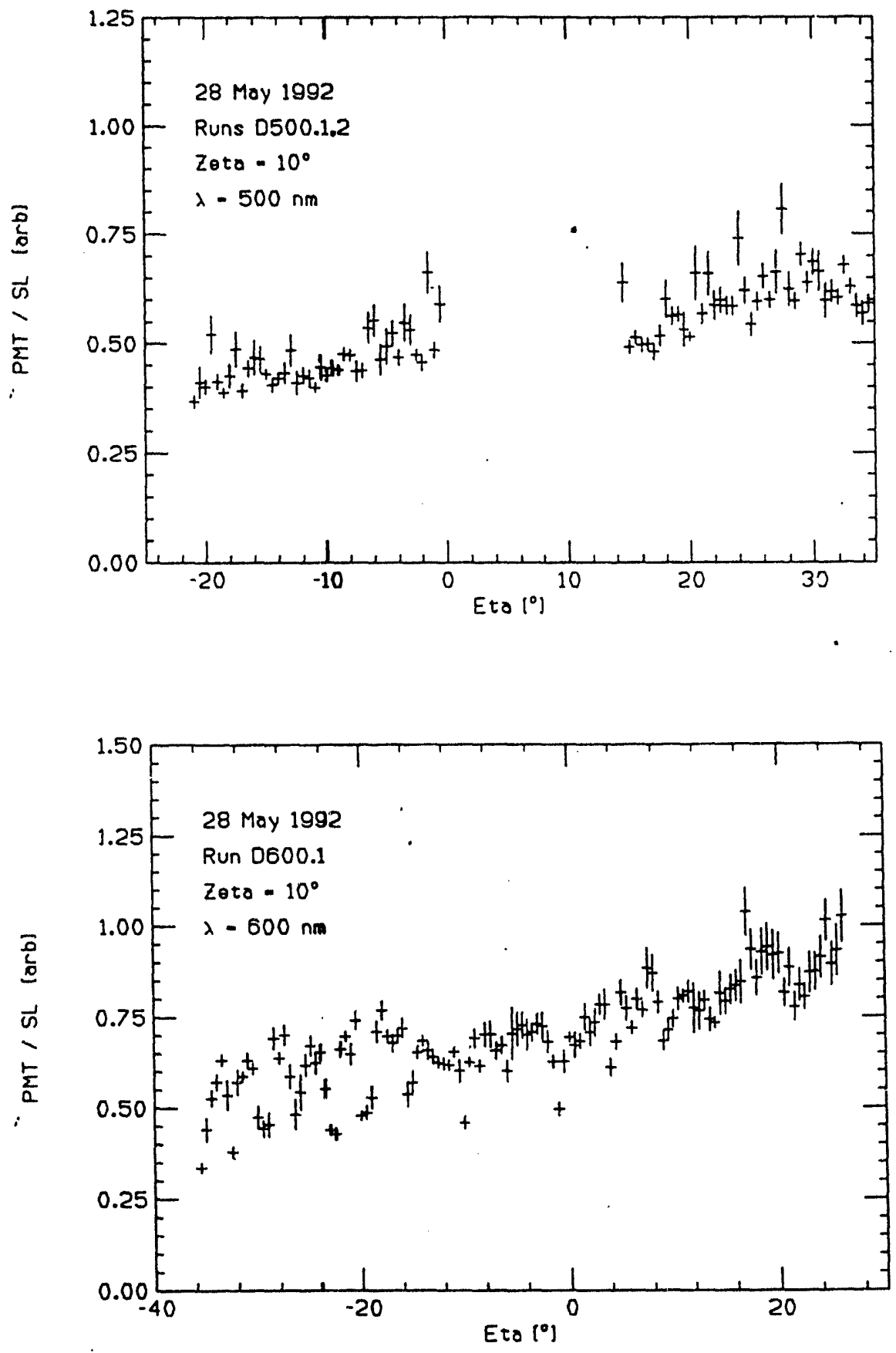

Fig. 13 Normalized PMT signal from the commercial grating as a function of the angle $\eta$ for radiation at 500 and $600 \mathrm{~nm}$. 

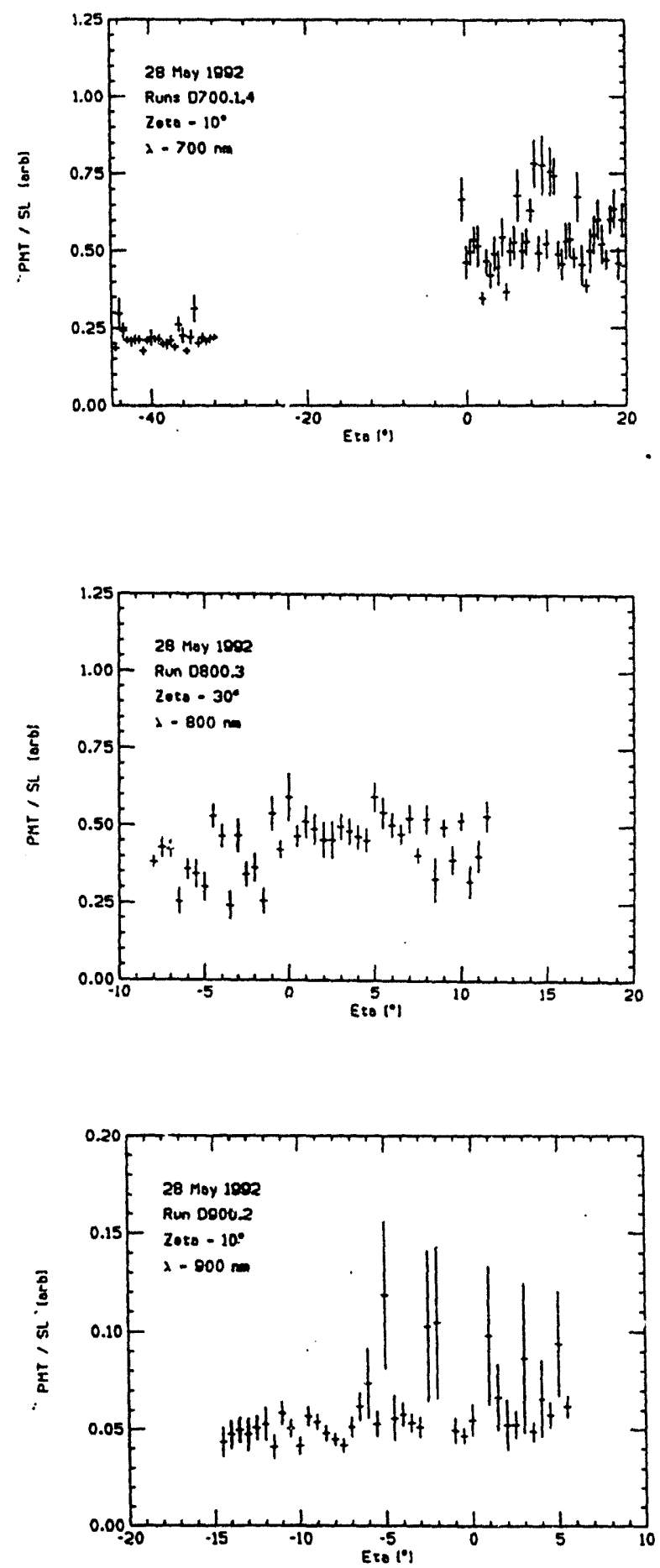

Fig. 13 (continued) Normalized PMT signal from the commercial grating as a function of the angle $\eta$ for radiation at 700,800 and $900 \mathrm{~nm}$. 


\section{2 / VISIBLE SMITH-PURCELL EXPERIMENT}

The majority of the 1992 beam time was used for an examination of the radiation off the $10.6 \mu \mathrm{m}$ period grating. Each period of data taking was preceded by a series of calibration runs that attempted to optimize the experimental conditions. Examples of these are given in Figs. 14-16, which show how the Faraday cup, PMT, and stripline detector were affected by the vertical trim coil, horizontal trim coil, and last quadrupole. The stripline was used to normalize all data and correct for variations in beam intensity from pulse to pulse. The Faraday cup was used as a diagnostic of shifts in the vertical position of the beam. Fig. 17 shows some data where we looked to see if changes in Faraday cup signal (i.e. position of the electron beam at the experiment) were correlated with the energy or vertical position of the laser pulse on the cathode. Studies were also made of the effects due to the momentum slit width, PMT high voltage, and beam tunes.

Fig. 18 shows a typical example of a $\zeta$ scan. Fig. 19 shows $\eta$ scans for five wavelengths over the full angular range that we were able to measure. The spectrum increases in general as the angle goes from the backward to forward direction. The signal drops in the extreme forward and backward directions because of acceptance. Note that there is a large backwards peak at wavelengths larger than $\approx 500 \mathrm{~nm}$. We confirmed in several runs that the backward peak is indeed missing at $400 \mathrm{~nm}$. Presumably, this peak is due to some kind of scintillation process in the silicon.

Small ( $=10 \%$ ) variations are seen in the spectra in Fig. 19. Unfortunately, the $10 \mu \mathrm{m}$ grating has diffraction orders with spacings that can be as close as $2.2^{\circ}$ at $400 \mathrm{~nm}$ to $4.9^{\circ}$ at $900 \mathrm{~nm}$. In addition, we do not know a priori which orders have large radiation peaks. Thus the only approach to detecting Smith-Purcell radiation with this grating is (1) repeat the scans and see if the peaks occur at the same angle and (2) show that any repeated peak occurs at an angle predicted by Eqn. 1. Figs. 20-22 show examples where we did this at three wavelengths. There are no obvious candidate peaks. Any Smith-Purcell radiation produced in the experiment must be smaller than the ripples seen on these curves and therefore is still a factor of at least 10 to 20 smaller than the background radiation. 

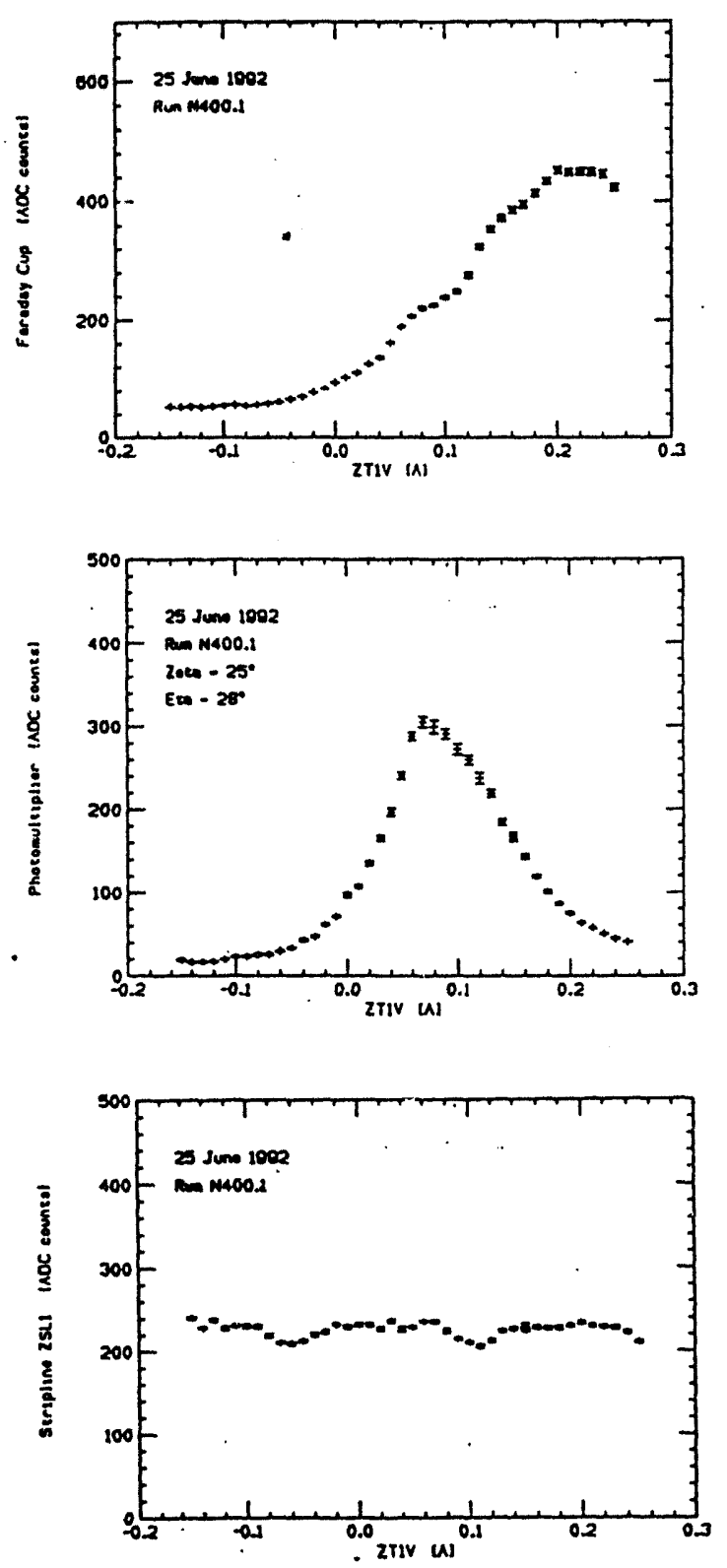

Fig. 14 Faraday cup, PMT, and stripline signals as a function of vertical trim current. 

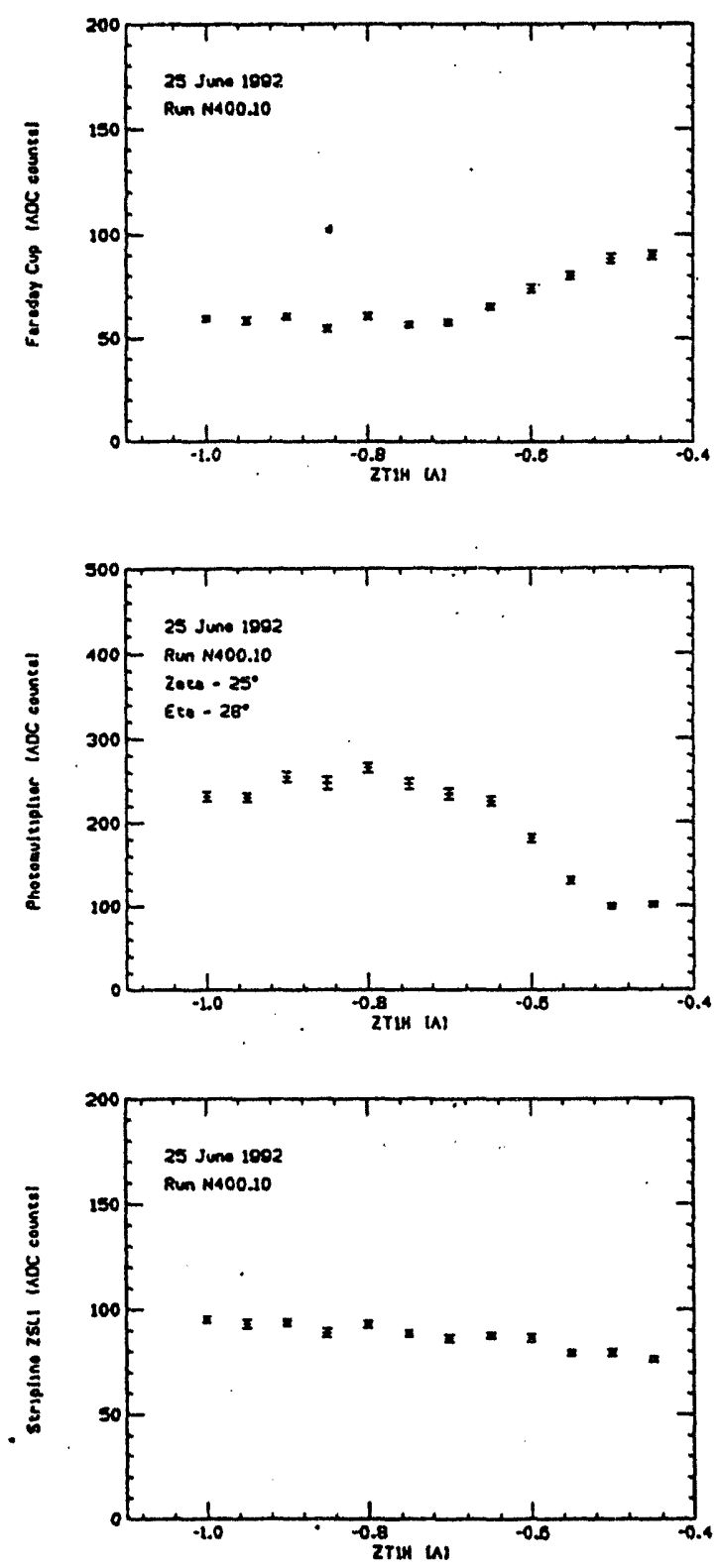

Fig. 15 Faraday cup, PMT, and stripline signals as a function of horizontal trim current. 

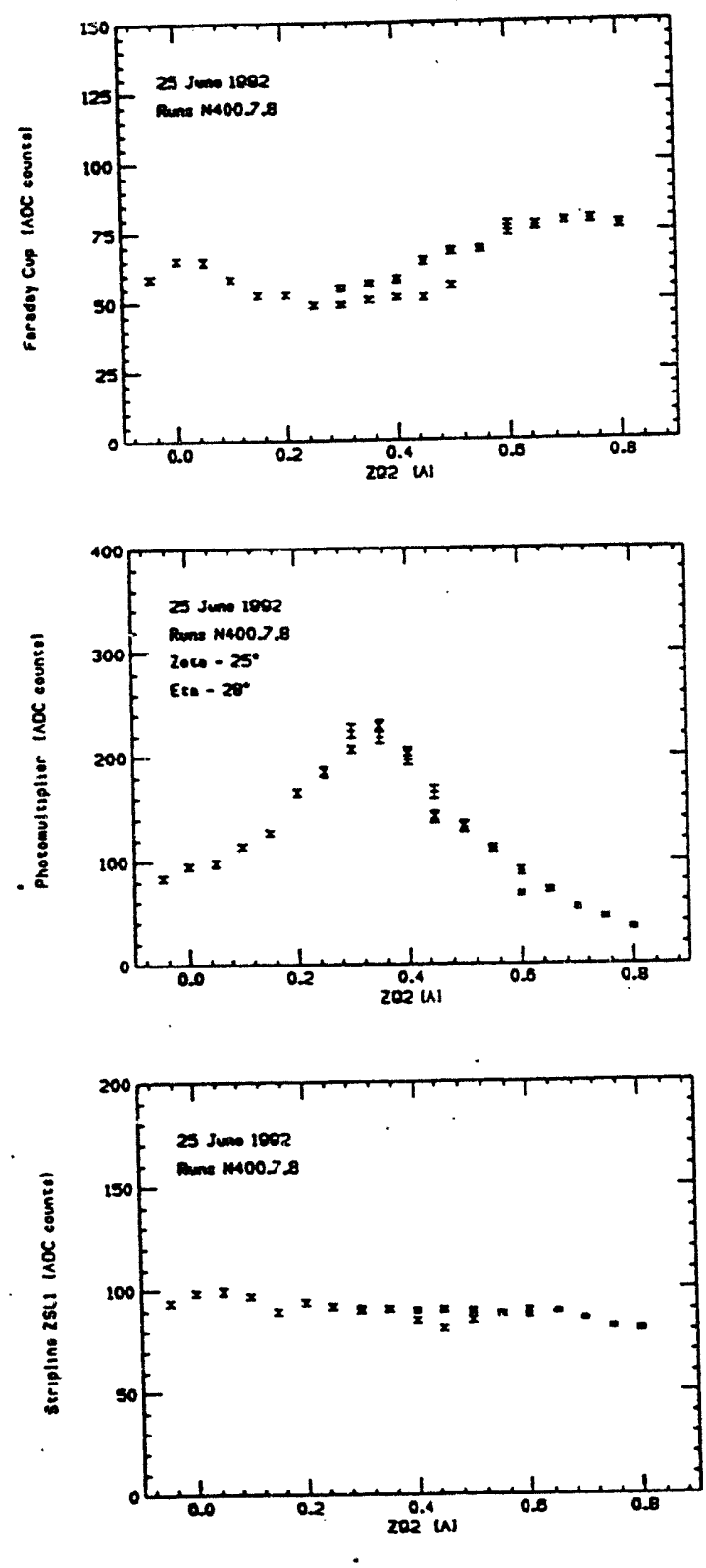

Fig. 16 Faraday cup, PMT, and stripline signals as a function of the current in the last beamline quadrupole. 

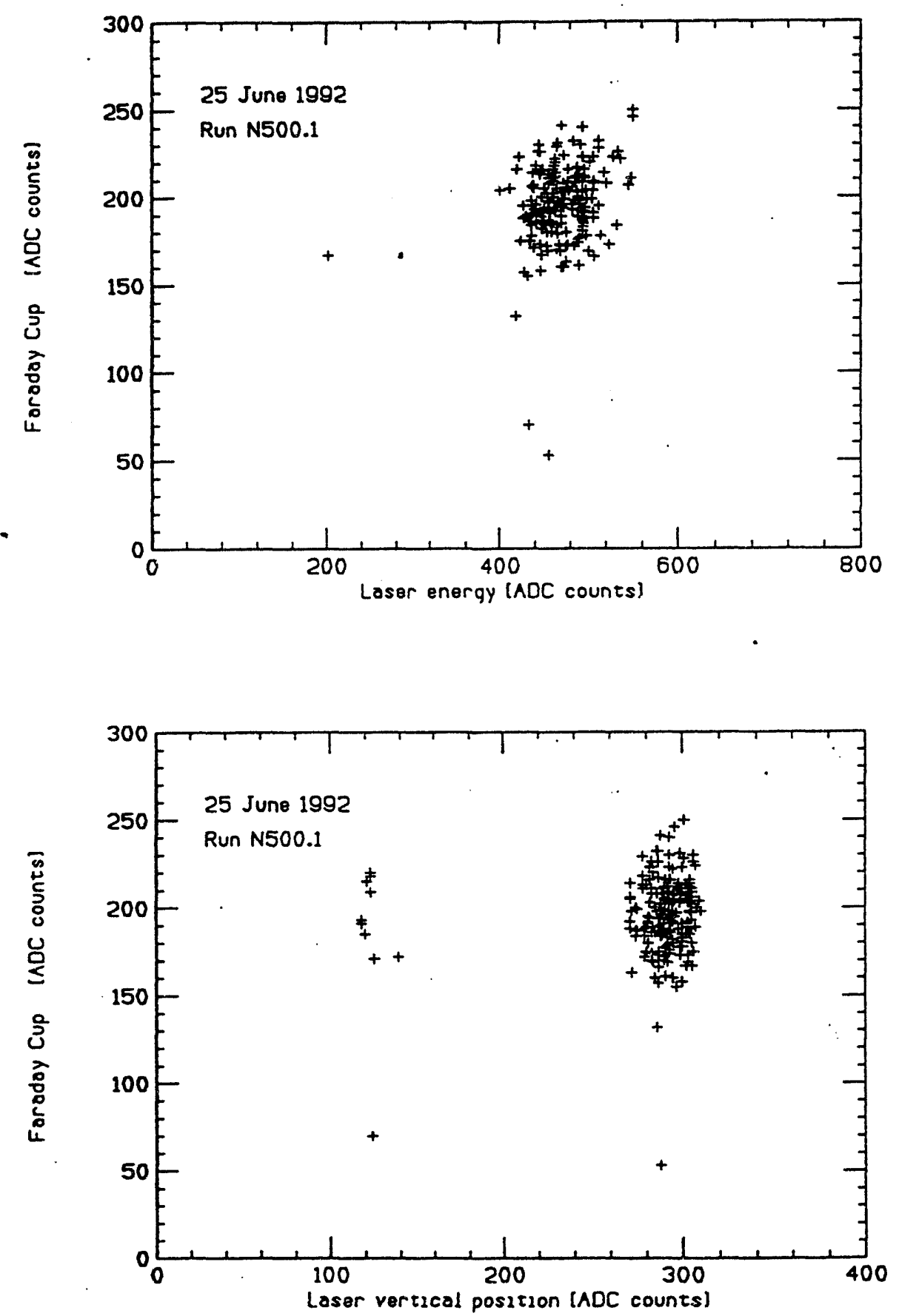

Fig. 17 Shot to shot correlation of the Faraday cup signal with the laser energy (from joulemeter) and laser vertical position on the cathode (from Spiricon). 


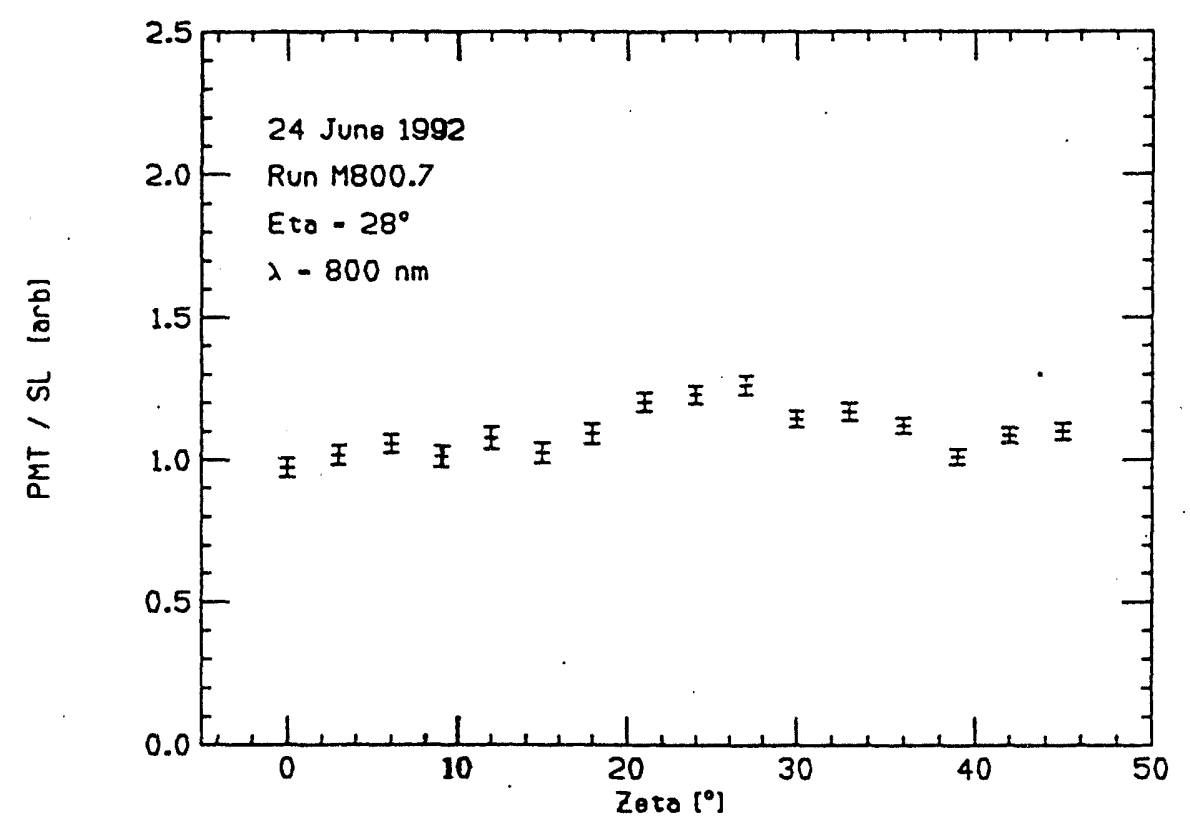

Fig. 18 Typical scan of normalized PMT signal as a function of the angle $\zeta$. 
28 / VISIBLE SMITH-PURCELL EXPERIMENT
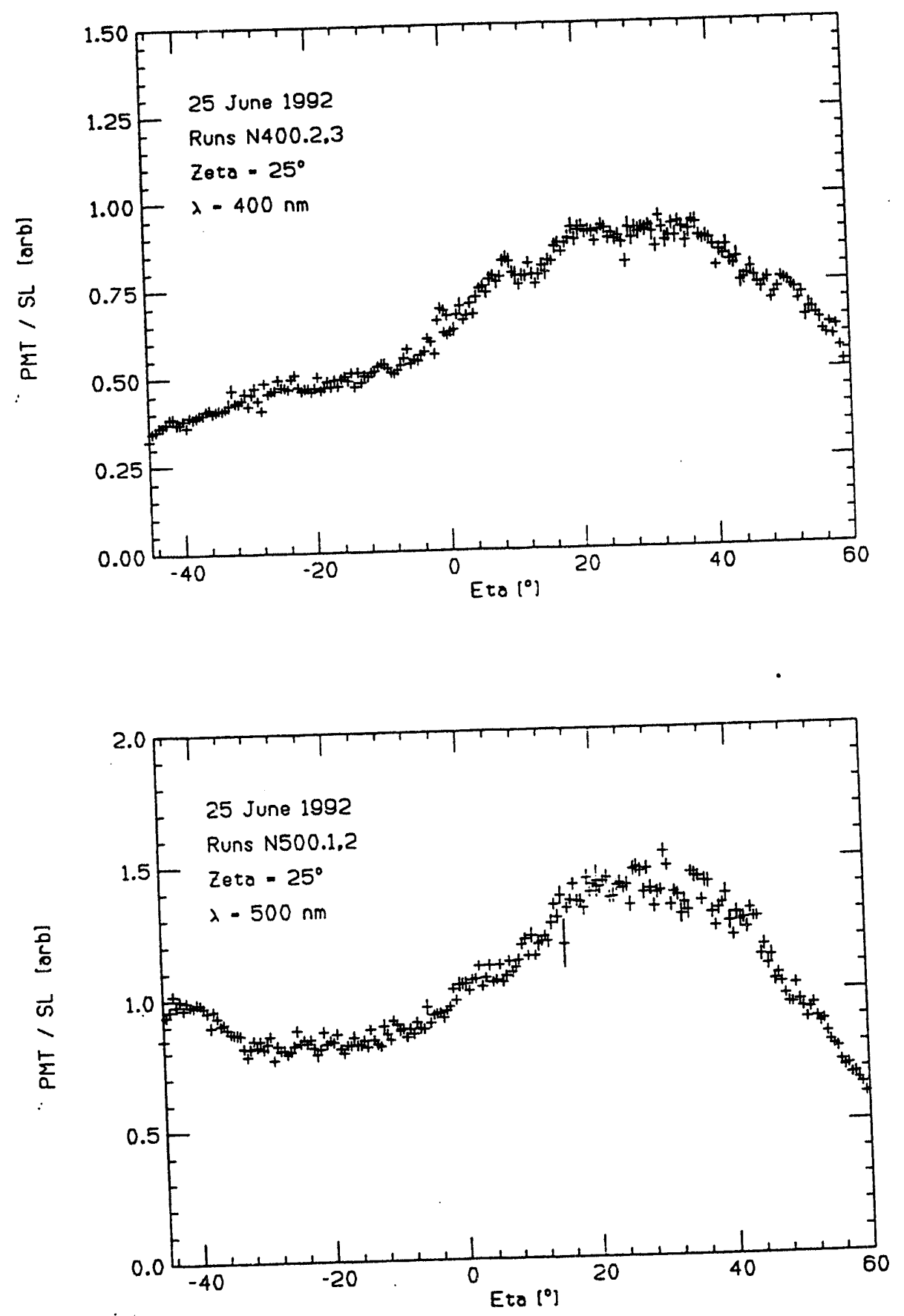

Fig. 19 Normalized PMT signal as a function of the angle $\eta$ for radiation at 400 and $500 \mathrm{~nm}$. 

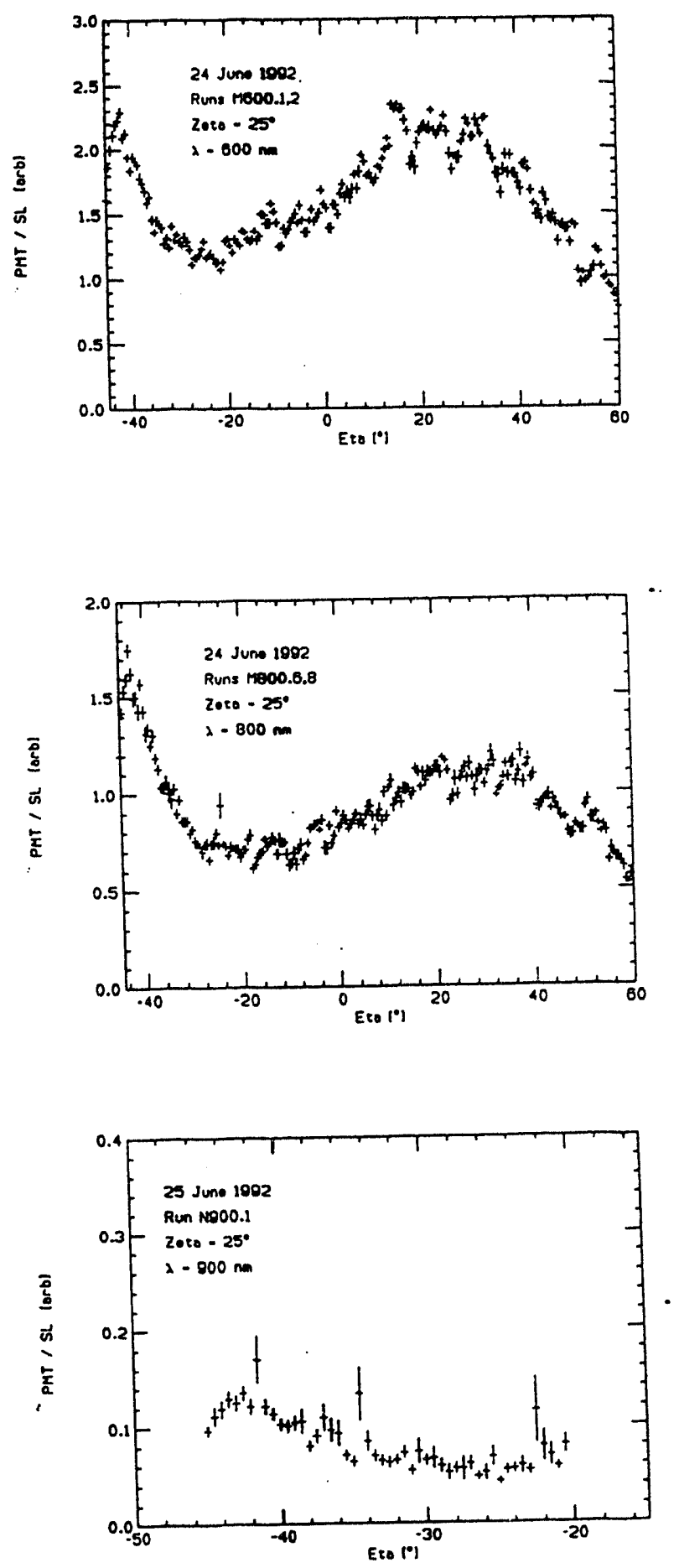

Fig. 19 (continued) Normalized PMT signal as a function of the angle $\eta$ for radiation at 600,800 and $900 \mathrm{~nm}$. 

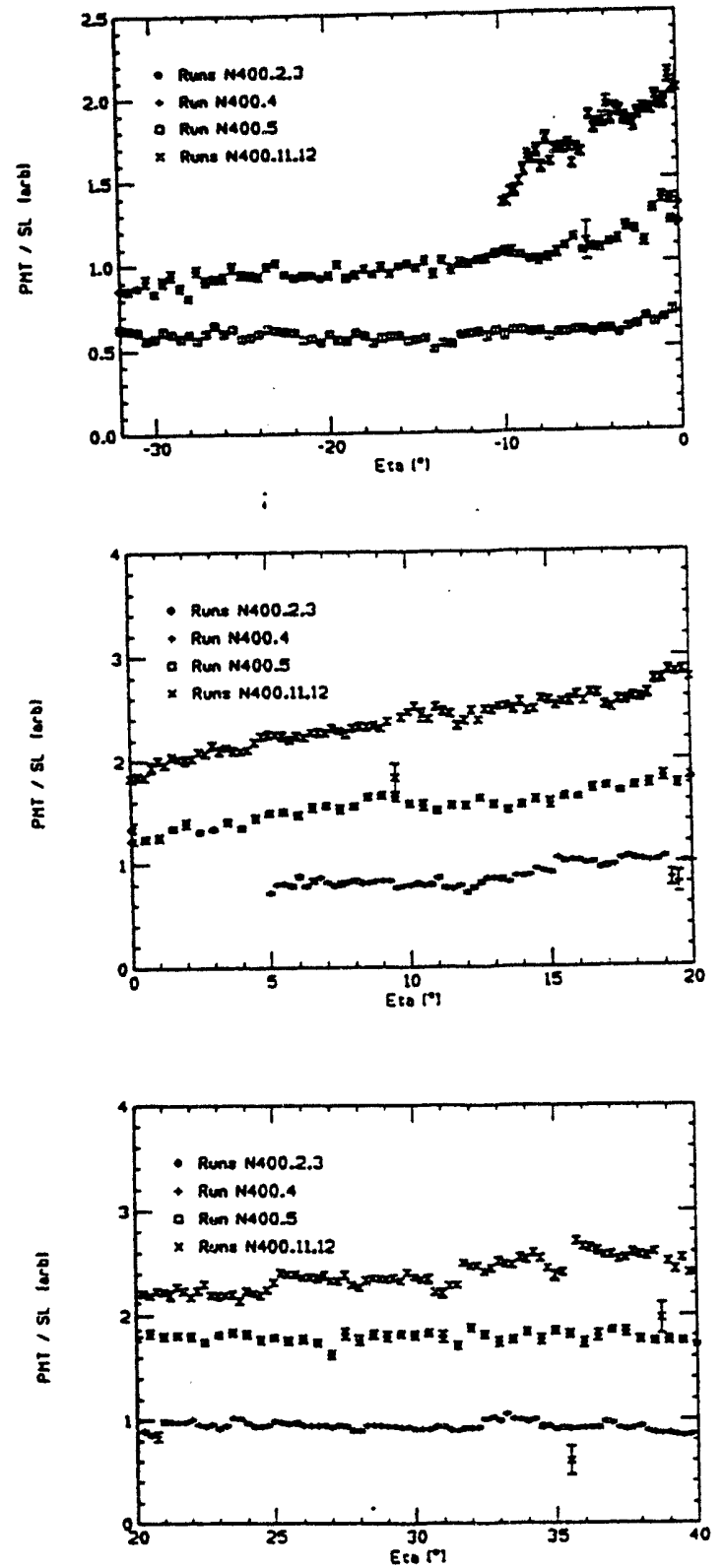

Fig. 20 Detailed comparison of normalized PMT signal as a function of the angle $\eta$ for $400 \mathrm{~nm}$ radiation. 

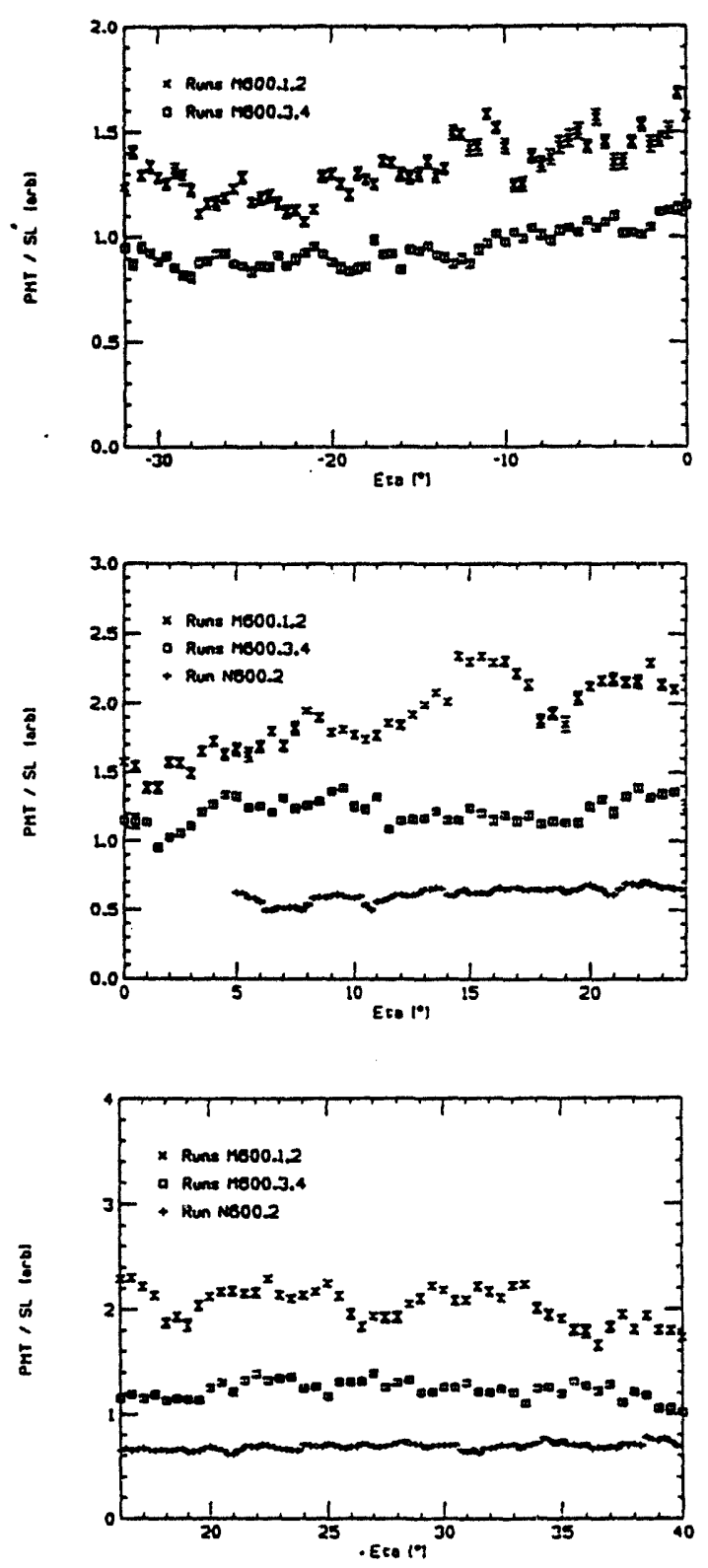

Fig. 21 Detailed comparison of normalized PMT signal as a function of the angle $\eta$ for $600 \mathrm{~nm}$ radiation. 

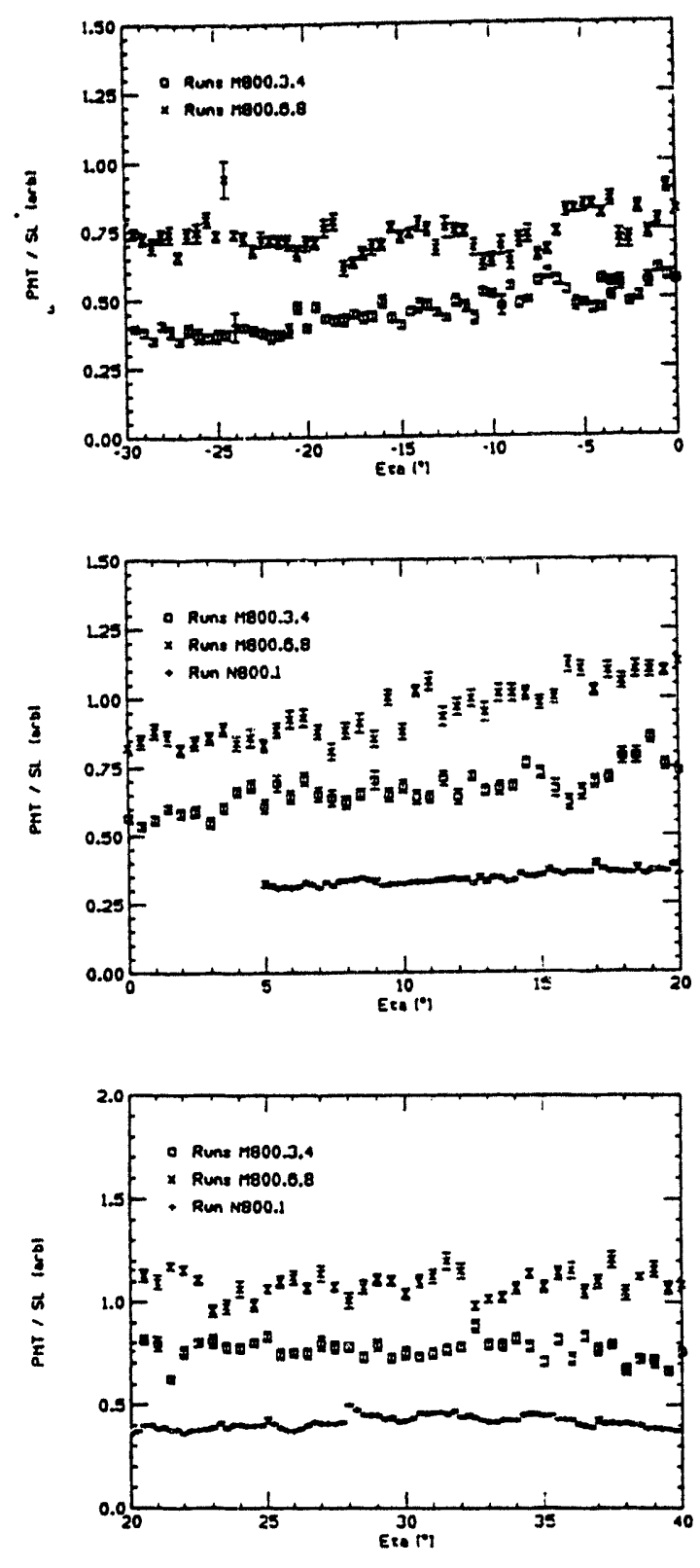

Fig. 22 Detailed comparison of normalized PMT signal as a function of the angle $\eta$ for $800 \mathrm{~nm}$ radiation. 


\section{Conclusions}

The obvious conclusion is that measuring visible Smith-Purcell radiation with ATF beams is difficult. At least two other laboratories [9] have attempted to measure visible radiation from multi-MeV electron beams and, at least so far, both have falled. To date the shortest wavelength radiation observed at the ATF has been $\approx 650 \mu \mathrm{m}$ in the Dartmouth experiment [10].

If someone were inclined to try this experiment again, we make the following recommendations based on our experience.

(1) Use a beamline with collimation and magnetic bending upstream of the experiment. The profile of the beam coming over the grating should be as sharp as possible. Avoid having the beam strike the grating.

(2) House the experiment in a high vacuum chamber, so that windows and glass tubes are avoided.

(3) Keep the detector as shielded as possible and outside the chamber.

(4) Much of the background radiation is certainly coming from transition radiation and bremsstrahlung. It would be useful if the experiment could do some background runs to specifically measure the spectra from these processes. If the beam conditions were extremely stable and very well monitored, it might be possible to make meaningful background corrections.

(5) Use a metal master grating (not a replica grating). This will alleviate worries about damage to the grating surface.

(6) Keep the grating on a vertical translation stage.

(7) Put a beam flag inside the chamber at the grating location so that the beam profile can be checked when desired.

(8) Use stepping motors for positioning. These are operationally much more convenient than the DC motors used in the Dartmouth experiment.

(9) The dual rotating stages were very convenient for mapping the radiation spectrum over a large solid angle.

(10) The CAMAC data acquisition with gated ADCs worked well and was convenient for sampling and correlating many signals. The GPIB system used in the Dartmouth experiment is convenient for storing quantities like scope traces. 


\section{4 / VISIBLE SMITH-PURCELL EXPERIMENT}

The adoption of all these points would lead to a considerably more expensive apparatus than was used in this experiment, but it may be necessary in order to measure the effect.

\section{Acknowledgements}

We would like to thank Joe Rogers, Leah Strichartz, John Warren and Doug Petkie for their help on this experiment. We would like to thank Kirk MCDonald and Bob Palmer for useful discussions.

\section{Notes References}

[1] S. Smith \& E. Purcell, Visible light from localized surface charges moving across a grating, Phys. Rev. 92:1069, 1953.

[2] A. Gover et al, Angular radiation pattern of Smith-Purcell radiation, J. Opt. Soc. Am. B1:723-8, 1984 .

[3] P. van den Berg, Smith-Purcell radiation from a point charge moving parallel to a reflection grating, J. Opt. Soc. Am. 63:1588$97,1973$.

[4] R. Fernow et al, Proposal for a Smith-Purcell experiment using the ATF thermionic gun, informal report RCF-LL-8703, 1987.

[5] R. Fernow, Does Lawson's theorem apply to Smith-Purcell radiation?, informal report $\mathrm{RCF}-\mathrm{AA}-9210$, 1992.

[6] $\mathrm{X}$. Wang, The Smith-Purcell radiation experiment, CAP-ATF technical note \#6.

[7] R. Fernow, Design of a grating for studying Smith-Purcell radiation and electron acceleration, in C. Joshi (ed), Advanced Accelerator Concepts, AIP Conf. Proc. 193:87-103, 1989.

[8] R. Fernow, Expected signal for the Smith-Purcell experiment, informal report RCF-AA-9104, 1991.

[9] Argonne and Rossendorf; private communications.

[10] One piece of anecdotal information from the Dartmouth experiment is illuminating. One day we happened to look at a $4 \mathrm{~mm}$ period grating with the TV camera. A bright glow was visible around the grating when the beam was on! visible background radiation processes from multi-MeV electron beams are clearly formidable. 
Appendix A: Time line

Nov 1987

Jun 1988

Sep 1989

Jan 1991

5 Feb

25 Feb

14 Mar

20 Mar

28 Mar

4 Apr

9 Apr

6 June

10 June

19 June

25 July

1992

$\begin{aligned} 20 & \text { May } \\ 1 & \text { June } \\ 22 & \text { June } \\ 25 & \text { June }\end{aligned}$

Original proposal

Amended proposal

Experiment chamber and data acquisition ready

Set up apparatus on ATF floor

Observe PMT signal from dark current

First 1991 data scan

Begin measuring scraper charge as diagnostic

Begin using $400 \mathrm{~nm}$ optical filter

Begin using $z$ line stripline as diagnostic

$Z$ line is resurveyed and realigned

Rechecked beam profile at grating location

First data run with photocurrent

Installed grating with sides painted black

First data run with supercharge

Measured PMT pick up of $x$ rays outside chamber Last run ror 1991.

Data run with modified arrangement

First beam. Using commercial grating

Change to $10 \mu \mathrm{m}$ period grating

First run with grating repositioned after laser alignment

Last data run 

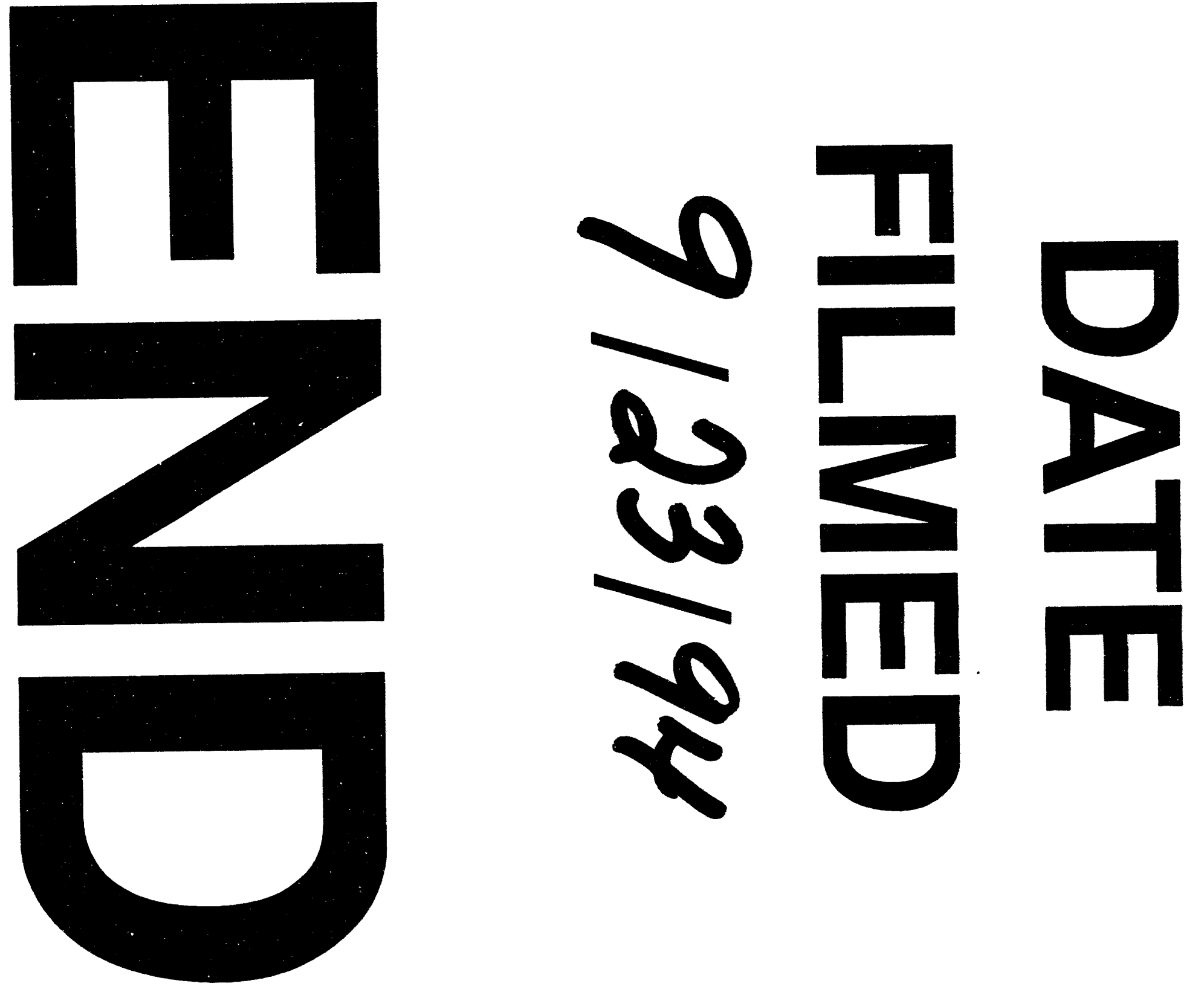

L 